Homology, Homotopy and Applications, vol. 8(1), 2006, pp.211-242

\title{
CODESCENT THEORY II: COFIBRANT APPROXIMATIONS
}

\author{
PAUL BALMER AND MICHEL MATTHEY*
}

(communicated by D. Arlettaz)

\begin{abstract}
We establish a general method to produce cofibrant approximations in the model category $\mathcal{U}_{\mathcal{S}}(\mathcal{C}, \mathcal{D})$ of $\mathcal{S}$-valued $\mathcal{C}$-indexed diagrams with $\mathcal{D}$-weak equivalences and $\mathcal{D}$-fibrations. We also present explicit examples of such approximations. Here, $\mathcal{S}$ is an arbitrary cofibrantly generated simplicial model category, and $\mathcal{D} \subset \mathcal{C}$ are small categories. An application to the notion of homotopy colimit is presented.
\end{abstract}

\section{Introduction}

The present paper may be read independently of Part I.

It is an important problem to understand model structures on categories of diagrams $\mathcal{S}^{\mathcal{C}}$, indexed by a small category $\mathcal{C}$ and with values in some model category $\mathcal{S}$, like, for instance, the category of (compactly generated Hausdorff) topological spaces. One recent illustration of this importance, among many others, is given in [2], where we show that the $K$-theoretic isomorphism conjectures boil down to understanding cofibrant approximations in a suitable category of diagrams. In this spirit, cofibrant approximations might be thought of as global assembly maps.

For an arbitrary model category $\mathcal{S}$, there is in general no known model structure on $\mathcal{C}$-indexed diagrams $\mathcal{S}^{\mathcal{C}}$, with objectwise weak equivalences. Hence the notion of "model approximation" of Chachólski and Scherer [4], which is not used here. Nevertheless, it is well known that if the model category $\mathcal{S}$ is cofibrantly generated, one can create a so-called left model structure on $\mathcal{S}^{\mathcal{C}}$, denoted $\mathcal{U}_{\mathcal{S}}(\mathcal{C})$ hereafter, by defining the weak equivalences and the fibrations $\mathcal{C}$-objectwise and by forcing the cofibrations by a left lifting property. In Part I, we even needed a $\mathcal{D}$-relative model structure on $\mathcal{S}^{\mathcal{C}}$, which we have denoted $\mathcal{U}_{\mathcal{S}}(\mathcal{C}, \mathcal{D})$, where the weak equivalences and the fibrations are defined $\mathcal{D}$-objectwise only, on a subset of objects $\mathcal{D} \subset \mathcal{C}$, see $[\mathbf{1}$, Theorem 3.5]. The reader of Dugger [5] and Hirschhorn [9] can as well keep the absolute case $\mathcal{D}=\mathcal{C}$ in mind.

Unfortunately, these left model constructions, although very popular, usually leave the cofibrations mysterious. The factorization axiom in $\mathcal{S}^{\mathcal{C}}$, which guarantees their abundance, generally roots back to Quillen's small object argument and this makes it hard to control what cofibrant approximations are. To be on the safe side,

* Deceased

Received February 19, 2005, revised September 12, 2005; published on December 22, 2005.

2000 Mathematics Subject Classification: 18G55; 55U10.

Key words and phrases: diagram category, cofibrant approximation, codescent.

Copyright (c) 2005, International Press. Permission to copy for private use granted. 
recall the terminology: a weak equivalence: $\xi: \mathcal{Q} X \longrightarrow X$ with $\mathcal{Q} X$ cofibrant is called a cofibrant approximation of $X$.

In this paper, assuming that $\mathcal{S}$ is a cofibrantly generated simplicial model category, we give an explicit construction of cofibrant approximations in categories of $\mathcal{S}$-valued diagrams. We do this in the relative case $\mathcal{U}_{\mathcal{S}}(\mathcal{C}, \mathcal{D})$ as well, mainly because we need it in $[2]$.

Of course, for $\mathcal{C}$ reduced to a point, $\mathcal{S}^{\mathcal{C}}$ is nothing but $\mathcal{S}$ and there is no hope that a general process for $\mathcal{C}$-indexed diagrams suddenly provides us with new cofibrant approximations in an arbitrary $\mathcal{S}$. Therefore, our method focusses on the "diagrammatic part" of the story and we consider cofibrant approximations in $\mathcal{S}$ itself as being under control. This cofibrant approximation in the category $\mathcal{S}$ could even be the identity if everybody is cofibrant in $\mathcal{S}$, like, e.g. in the category sSets of simplicial sets.

Let $X \in \mathcal{S}^{\mathcal{C}}$ be a diagram. Let us start looking for a cofibrant approximation $\xi: Y \rightarrow X$. The first observation is that a cofibrant diagram $Y \in \mathcal{S}^{\mathcal{C}}$ is always objectwise cofibrant, i.e. $Y(c)$ is cofibrant in $\mathcal{S}$ for all $c \in \mathcal{C}$. So, it cannot harm to first replace $X$ objectwise by a functorial cofibrant approximation in $\mathcal{S}$. We produce in this way a first cheap approximation $\eta: q X \rightarrow X$, where $q X(c)=\mathcal{Q}_{\mathcal{S}}(X(c))$. Although $q X$ is objectwise cofibrant and the map $\eta$ is a $\mathcal{C}$-weak equivalence, this $q X$ is in general not cofibrant as a diagram! So far, we have done as much as we could do just using the category of values $\mathcal{S}$. We now need to turn to the internal structure of the index-category $\mathcal{C}$ and, in the relative case, of the subcategory $\mathcal{D} \subset \mathcal{C}$.

Recall that a simplicial model category (see Appendix A) is in particular equipped with an action $\odot:$ sSets $\times \mathcal{S} \longrightarrow \mathcal{S}$ of the category of simplicial sets. This can be jazzed up into a "tensor product"

$$
-\stackrel{\mathcal{Q}}{\mathcal{D}}_{\mathcal{D}}-: \quad \mathrm{sSets}^{\mathcal{D}^{\mathrm{op}} \times \mathcal{C}} \times \mathcal{S}^{\mathcal{D}} \longrightarrow \mathcal{S}^{\mathcal{C}} .
$$

This is probably well known to the experts but we shall explain this carefully in Section 3.

Our point is that finding cofibrant approximations of $\mathcal{C}$-indexed diagrams with values in $\mathcal{S}$ amounts to finding one cofibrant approximation of one very special and canonical diagram $\mathbb{F}$, living in $\mathcal{S S e t s}^{\mathcal{D}^{\mathrm{op}} \times \mathcal{C}}$ and described below, and then to tensor it with any object we want to cofibrantly approximate. In some sense, this diagram $\mathbb{F}$ encodes the purely $(\mathcal{C}, \mathcal{D})$-part of the problem. We state the following main result in relative form and then unfold the case $\mathcal{C}=\mathcal{D}$.

Theorem 1.1. Let $\mathcal{C}$ be a small category and let $\mathcal{D} \subset \mathcal{C}$ be a full subcategory. Define $\mathbb{F} \in \mathrm{sSets}^{\mathcal{D}^{\mathrm{op}} \times \mathcal{C}}$ as follows: for any $d \in \mathcal{D}$ and $c \in \mathcal{C}$,

$$
\mathbb{F}(d, c):=\operatorname{mor}_{\mathcal{C}}(d, c) \quad \in \text { sSets }
$$

where the set $\operatorname{mor}_{\mathcal{C}}(d, c)$ is seen as a constant (or discrete) simplicial set. Choose one cofibrant approximation of $\mathbb{F}$ in $\mathcal{U}_{\mathrm{s} S \mathrm{Sets}}\left(\mathcal{D}^{\mathrm{op}} \times \mathcal{C}, \mathcal{D}^{\mathrm{op}} \times \mathcal{D}\right)$, say

$$
\mathbb{E} \longrightarrow \mathbb{F} \text {. }
$$


Let now $\mathcal{S}$ be a cofibrantly generated simplicial model category and let $X \in \mathcal{S}^{\mathcal{C}}$ be a diagram. Let $q X \rightarrow X$ be an objectwise cofibrant approximation of $X$. Then,

$$
\xi_{X}: \quad \mathbb{E} \stackrel{\mathcal{C}}{\bigotimes_{\mathcal{D}}} \operatorname{res}_{\mathcal{D}}^{\mathcal{C}}(q X) \longrightarrow X
$$

is a cofibrant approximation of $X$ in the model category $\mathcal{U}_{\mathcal{S}}(\mathcal{C}, \mathcal{D})$. In particular, if $X \in \mathcal{S}^{\mathcal{C}}$ is objectwise cofibrant, then $\mathbb{E} \stackrel{\mathcal{D}}{\otimes}_{\mathcal{C}} \operatorname{res}_{\mathcal{D}}^{\mathcal{C}} X \longrightarrow X$ is a cofibrant approximation of $X$ in $\mathcal{U}_{\mathcal{S}}(\mathcal{C}, \mathcal{D})$. The morphism $\xi_{X}$ is the "obvious" one (see Theorem 5.4).

We shall need in $[\mathbf{2}]$ the above generality. However, since the absolute case $\mathcal{D}=\mathcal{C}$ widely predominates in the literature, we now unfold the above theorem in this situation; moreover, the morphism $\xi_{X}$ then becomes quite explicit.

Corollary 1.2. Let $\mathcal{C}$ be a small category. Consider $\mathbb{F} \in \mathrm{sSets}^{\mathcal{C}^{\mathrm{op}} \times \mathcal{C}}$, defined for any $c^{\prime}, c \in \mathcal{C}$ by $\mathbb{F}\left(c^{\prime}, c\right):=\operatorname{mor}_{\mathcal{C}}\left(c^{\prime}, c\right) \in \mathrm{s} \mathcal{S}$ ets (simplicially constant). Choose a cofibrant approximation $\vartheta: \mathbb{E} \longrightarrow \mathbb{F}$ of $\mathbb{F}$ in the left model structure on $\mathrm{s} \operatorname{ets}^{\mathcal{C}^{\mathrm{op}} \times \mathcal{C}}$. Let $\mathcal{S}$ be a cofibrantly generated simplicial model category and $X \in \mathcal{S}^{\mathcal{C}}$ a diagram. Let $\eta: q X \rightarrow X$ be an objectwise cofibrant approximation of $X$. Then the composite

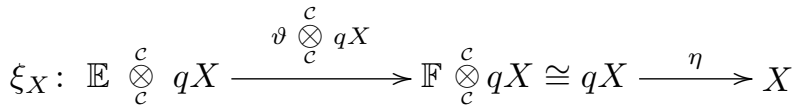

is a cofibrant approximation of $X$ in the left model category structure on $\mathcal{S}^{\mathcal{C}}$.

The customer is now entitled to ask for an explicit cofibrant approximation $\mathbb{E} \rightarrow \mathbb{F}$ of the object $\mathbb{F} \in \mathrm{sSets}^{\mathcal{D}^{\mathrm{op}} \times \mathcal{C}}$ of Theorem 1.1 with respect to the relative model structure $\mathcal{U}_{\text {s } S \text { ets }}\left(\mathcal{D}^{\text {op }} \times \mathcal{C}, \mathcal{D}^{\text {op }} \times \mathcal{D}\right)$. Here it comes (see Theorem 6.5.).

Theorem 1.3. Let $\mathcal{C}$ be a small category and let $\mathcal{D} \subset \mathcal{C}$ be a full subcategory. Define $\mathbb{F} \in \operatorname{sSets}^{\mathcal{D}^{\mathrm{op}} \times \mathcal{C}}$ as above, i.e. $\mathbb{F}(d, c)=\operatorname{mor}_{\mathcal{C}}(d, c) \in \mathrm{s} \mathcal{S e t s}$ (simplicially constant). Define the functor $\mathbb{E} \in \mathrm{sSets}^{\mathcal{D}^{\mathrm{op}} \times \mathcal{C}}$ as follows. For $d \in \mathcal{D}$ and $c \in \mathcal{C}$, put

$$
\mathbb{E}(d, c):=B(d \searrow \mathcal{D} \searrow c)^{\mathrm{op}}
$$

with $B$ standing for the usual nerve of the category $(d \searrow \mathcal{D} \searrow c)^{\mathrm{op}}$; here, $(d \searrow \mathcal{D} \searrow c)$ is the "comma" category of triples $(\alpha, x, \beta)$, where $x \in \mathcal{D}$ and where $\alpha: d \rightarrow x$ and $\beta: x \rightarrow c$ are arrows in $\mathcal{C}$, the morphisms in $(d \searrow \mathcal{D} \searrow c$ ) are the obvious ones (see Notation 6.1). Consider the morphism $\vartheta: \mathbb{E} \longrightarrow \mathbb{F}$ given for $d \in \mathcal{D}$ and $c \in \mathcal{C}$ by the map

$$
\vartheta(d, c): \quad \mathbb{E}(d, c)=B\left(d \searrow \mathcal{D} \searrow^{\mathcal{c}}\right)^{\mathrm{op}} \longrightarrow \operatorname{mor}_{\mathcal{C}}(d, c)=\mathbb{F}(d, c),
$$

which is the evident "composition of everything" in each simplicial degree. Then, $\vartheta: \mathbb{E} \longrightarrow \mathbb{F}$ is a cofibrant approximation of $\mathbb{F}$ in $\mathcal{U}_{\mathrm{s} S e t s}\left(\mathcal{D}^{\mathrm{op}} \times \mathcal{C}, \mathcal{D}^{\mathrm{op}} \times \mathcal{D}\right)$.

Similarly, there is a cofibrant approximation ${ }^{\natural} \vartheta:{ }^{\natural} \mathbb{E} \longrightarrow \mathbb{F}$, where the object ${ }^{\natural} \mathbb{E}$ is defined as above but without "op", that is, ${ }^{\natural} \mathbb{E}(d, c):=B(d \searrow \mathcal{D} \searrow c)$. 
Combining Theorems 1.1 and 1.3, we obviously get explicit cofibrant approximations in $\mathcal{U}_{\mathcal{S}}(\mathcal{C}, \mathcal{D})$, see Corollary-Definition 6.6. Let us stress the universal character of these results. Mastering one cofibrant approximation of one particular diagram $\mathbb{F}$ taking values in simplicial sets yields cofibrant approximations in $\mathcal{C}$-indexed diagrams with values in arbitrary reasonable model categories. Some authors, who like to think, and to use, that whatever holds for diagrams in sSets remains true for diagrams in familiar model categories, now have a rigorous argument at their disposal.

As already observed in Hollender and Vogt [12] in the special case of topological spaces, the above diagram $\mathbb{E}$ is of central importance for homotopy colimits. The last short section of the paper is an application of the above to a question regarding homotopy colimits that many topologists might have asked themselves once. Two approaches to homotopy colimits are available, both originating from the work of Bousfield and Kan [3]. First, one can think of the homotopy colimit basically as an esoteric but explicit formula which one can apply to whatever moves around, say, in any category with an action of simplicial sets. The second approach, slightly more conceptual, prefers to dwell on the problem that good old colimits do not preserve weak equivalences, whereas homotopy colimits should ideally preserve them. Homotopy colimits would therefore be better suited for homotopy theory. In a more model theoretical language, the homotopy colimit should be thought of as the left derived functor of the colimit. Such a definition makes better sense if the category of diagrams is a model category, like, for instance, if $\mathcal{S}$ is a cofibrantly generated model category. When both approaches make sense, namely if $\mathcal{S}$ is a cofibrantly generated simplicial model category, as we consider here, it is reasonable and legitimate to ask whether both notions coincide, i.e. are weakly equivalent. The obvious obstruction is that the standard homotopy colimit (the first mentioned above), if it ends up being homotopy equivalent to a left derived functor, will itself preserve weak equivalences. This is in fact the one and only obstruction, as explained in Theorem 7.2 and Remark 7.4, which in particular implies the following result.

Theorem 1.4. Let $\mathcal{S}$ be a cofibrantly generated simplicial model category and let $\mathcal{C}$ be a small category. Assume that hocolim $\mathcal{C}_{\mathcal{C}}: \mathcal{S}^{\mathcal{C}} \longrightarrow \mathcal{S}$ takes $\mathcal{C}$-weak equivalences to weak-equivalences. Then there is a natural zig-zag of two weak equivalences

$$
\underset{\mathcal{C}}{\operatorname{hocolim}} X \sim \underset{\mathcal{C}}{\operatorname{colim}} X
$$

for any $X \in \mathcal{S}^{\mathcal{C}}$, where $\operatorname{Lcolim}_{\mathcal{C}} X$ is any left derived functor of $\operatorname{colim}_{\mathcal{C}}$, that is, Lcolim $_{\mathcal{C}}=\operatorname{colim}_{\mathcal{C}} \circ \mathcal{Q}$ where $\mathcal{Q}$ is any functorial cofibrant approximation in the category $\mathcal{S}^{\mathcal{C}}$ with the absolute model structure $\mathcal{U}_{\mathcal{S}}(\mathcal{C})$. Moreover, for $\mathcal{Q}$ suitably constructed with the methods of Corollary 1.2 and Theorem 1.3, this zig-zag of weak equivalences reduces to a single natural weak equivalence $\operatorname{hocolim}_{\mathcal{C}} X \stackrel{\sim}{\longrightarrow} L \operatorname{colim}_{\mathcal{C}} X$.

The organization of the article should be clear from this introduction. Let us simply add that we need some flexibility in "couplings" like the $\odot$ or the $\stackrel{\mathcal{D}}{\mathcal{D}}_{\text {considered }}$ coter above and that this is better understood when abstracted a bit into a general notion of coupling $\mathcal{S}_{1} \times \mathcal{S}_{2} \longrightarrow \mathcal{S}_{3}$, with three possibly different categories involved. This 
gives us a fair chance to understand who does what in the subsequent constructions. This formalism is developed in Sections 3 and 4.

Remark 1.5. It is likely that the same results as those obtained here can also be deduced, in a different manner, from Dugger's article [5], but we did not pursue in this direction. Several special cases of our constructions are already available in the literature, like in the very complete [9], for instance. Our main result which we use in the sequel [2], i.e. the explicit description of cofibrant approximations in the relative structure $\mathcal{U}_{\mathcal{S}}(\mathcal{C}, \mathcal{D})$, is new. We also hope that the reader will benefit from the systematic organization and from the relative concision of this article.

\section{Notations, diagram-categories and recollection on $\mathcal{U}_{\mathcal{S}}(\mathcal{C}, \mathcal{D})$}

This short section introduces the notations used in the sequel and presents some basic facts concerning categories of diagrams. It also contains a little summary of what is needed from Part I on the model category $\mathcal{U}_{\mathcal{S}}(\mathcal{C}, \mathcal{D})$.

We refer to Mac Lane's book [13] for purely categorical questions.

We refer to Hirschhorn [9] or to Hovey [10] for model category questions. Appendix A of Part I gives a concise list of prerequisites. The notion of simplicial model category being central here, it is recalled in Appendix A of the present part.

In a model category $\mathcal{M}$, recall the distinction between a cofibrant approximation of an object $X \in \mathcal{M}$, meaning a weak equivalence $\xi: \mathcal{Q} X \rightarrow X$ with $\mathcal{Q} X$ cofibrant, and the cofibrant replacement, the latter being the cofibrant approximation obtained from the functorial factorization axiom applied to the morphism $\varnothing \rightarrow X$; this means in particular that $\xi$ is also a fibration in the latter case.

We call a cofibrant approximation $(\mathcal{Q}, \xi)$ functorial if $\mathcal{Q}$ is a functor and if $\xi: \mathcal{Q} \longrightarrow \mathrm{id}_{\mathcal{M}}$ is a natural transformation. The cofibrant replacement is functorial and we sometimes designate it by $\left(Q_{\mathcal{M}}, \xi^{\mathcal{M}}\right)$.

Notation 2.1. Let $\mathcal{S}$ be a category and $\mathcal{C}$ a small category. Denote by $\mathcal{S}^{\mathcal{C}}$ the category of functors from $\mathcal{C}$ to $\mathcal{S}$, also called $\mathcal{S}$-valued $\mathcal{C}$-indexed diagrams. Write

$$
\mathrm{Cst}_{\mathcal{S}}^{\mathcal{C}}: \mathcal{S} \longrightarrow \mathcal{S}^{\mathcal{C}}, \quad s \longmapsto(\mathcal{C} \rightarrow \mathcal{S}, c \mapsto s)
$$

for the "constantification" functor; this way we can view $\mathcal{S}$ as a subcategory of $\mathcal{S}^{\mathcal{C}}$. We also write $\underline{s}$ for $\operatorname{Cst}_{\mathcal{S}}^{\mathcal{C}}(s)$. Note that the colimit functor colim ${ }_{\mathcal{C}}: \mathcal{S}^{\mathcal{C}} \longrightarrow \mathcal{S}$, when it exists, is left adjoint to the functor $\mathrm{Cst}_{\mathcal{S}}^{\mathcal{C}}$.

We denote by $\mathcal{S}$ ets the category of sets and by sets $=\mathcal{S e t s}^{{ }^{\mathrm{op}}}$ the category of simplicial sets.

Remark 2.2. The usual constantification functor $\mathrm{Cst}_{\mathcal{S} \text { ets }}^{\Delta^{\mathrm{op}}}$ : Sets $\longrightarrow$ s $\mathcal{S}$ ets has the following "level-zero functor" as right adjoint:

$$
(-)_{0}: \text { sSets } \longrightarrow \text { Sets, } \quad K=K_{\bullet} \longmapsto K_{0} \text { • }
$$

Convention 2.3. We denote a functor $F$ also as $F(-)$ or $F(?)$. If in some "formula" two functors $F$ and $G$ with the same source category $\mathcal{A}$ are involved, we write 
$F(?)$ and $G(?)$ to stress the fact that we evaluate $F$ and $G$ at the same dummyvariable object? of $\mathcal{A}$. We adopt similar notations with ?? and ??? in place of?, usually when several dummy-variables are involved.

Notation 2.4. For categories $\mathcal{S}, \mathcal{A}$ and $\mathcal{C}$, with $\mathcal{A}$ and $\mathcal{C}$ small, we make the following obvious identifications of categories of diagrams:

$$
\left(\mathcal{S}^{\mathcal{C}}\right)^{\mathcal{A}}=\mathcal{S}^{\mathcal{C} \times \mathcal{A}} \text { and }\left(\mathcal{S}^{\mathcal{A}}\right)^{\mathcal{C}}=\mathcal{S}^{\mathcal{A} \times \mathcal{C}}
$$

We denote the evident "switch functor" by $\sigma_{\mathcal{C}, \mathcal{A}}: \mathcal{S}^{\mathcal{C} \times \mathcal{A}} \stackrel{\cong}{\longrightarrow} \mathcal{A} \times \mathcal{C}$.

Definition 2.5. Let $\mathcal{S}$ be a cofibrantly generated model category and $\mathcal{D}$ a subcategory of a small category $\mathcal{C}$. A morphism $\varphi: X \longrightarrow Y$ in $\mathcal{S}^{\mathcal{C}}$ is a $\mathcal{D}$-isomorphism (resp. a $\mathcal{D}$-weak equivalence, a $\mathcal{D}$-fibration or a trivial $\mathcal{D}$-fibration) if for every object $d \in \mathcal{D}$, the morphism $\varphi(d): X(d) \longrightarrow Y(d)$ is an isomorphism (resp. a weak equivalence, a fibration or a trivial fibration) in $\mathcal{S}$.

Proposition-Definition 2.6. Let $\mathcal{S}$ be a cofibrantly generated model category, and $\mathcal{D}$ a subcategory of a small category $\mathcal{C}$. Then, there is a model category structure on the category $\mathcal{S}^{\mathcal{C}}$ of $\mathcal{C}$-indexed $\mathcal{S}$-valued diagrams, where the weak equivalences are the $\mathcal{D}$-weak equivalences and the fibrations are the $\mathcal{D}$-fibrations. It is denoted by $\mathcal{U}_{\mathcal{S}}(\mathcal{C}, \mathcal{D})$ and is called the $\mathcal{D}$-relative model structure on $\mathcal{S}^{\mathcal{C}}$. If a diagram $X \in \mathcal{S}^{\mathcal{C}}$ is cofibrant in $\mathcal{U}_{\mathcal{S}}(\mathcal{C}, \mathcal{D})$, we call it $\mathcal{D}$-cofibrant. When $\mathcal{D}=\mathcal{C}$, we also write $\mathcal{U}_{\mathcal{S}}(\mathcal{C})$ for $\mathcal{U}_{\mathcal{S}}(\mathcal{C}, \mathcal{C})$ and call it the absolute model structure on $\mathcal{S}^{\mathcal{C}}$.

See $\left[\mathbf{1}\right.$, Theorem. 3.5]. Note that the model category $\mathcal{U}_{\mathcal{S}}(\mathcal{C}, \mathcal{D})$ does not depend on the category structure of $\mathcal{D}$, but only on the underlying set of objects $\operatorname{obj}(\mathcal{D})$.

Remark 2.7. Let $\mathcal{C}$ be a small category, $\mathcal{D} \subset \mathcal{C}$ a subcategory and $\mathcal{S}$ a cofibrantly generated model category. It is proven in [1, Proposition 3.14] (see also Remark 8.8 therein) that if $f: X_{1} \longrightarrow X_{2}$ is a cofibration in $\mathcal{U}_{\mathcal{S}}(\mathcal{C}, \mathcal{D})$, then it is $\mathcal{C}$-objectwise a cofibration, that is, $f(c): X_{1}(c) \longrightarrow X_{2}(c)$ is a cofibration in $\mathcal{S}$ for each $c \in \mathcal{C}$. In particular, if $X \in \mathcal{U}_{\mathcal{S}}(\mathcal{C}, \mathcal{D})$ is a cofibrant diagram, then $X(c) \in \mathcal{S}$ is cofibrant as well, for every object $c \in \mathcal{C}$ (and not just $\mathcal{D}$-objectwise).

Recall that a category is (co)complete if it admits all small (co)limits.

Remark 2.8. Let $\mathcal{S}$ be a cocomplete category and $\mathcal{D}$ a subcategory of a small category $\mathcal{C}$. The restriction functor $\operatorname{res}_{\mathcal{D}}^{\mathcal{C}}$, from $\mathcal{S}^{\mathcal{C}}$ to $\mathcal{S}^{\mathcal{D}}$, has a left adjoint

$$
\operatorname{ind}_{\mathcal{D}}^{\mathcal{C}}: \mathcal{S}^{\mathcal{D}} \rightleftarrows \mathcal{S}^{\mathcal{C}}: \operatorname{res}_{\mathcal{D}}^{\mathcal{C}}
$$

called the induction functor (see, for instance, $[\mathbf{1}$, Appendix. B]). Assume now that $\mathcal{S}$ is a cofibrantly generated model category. It is clear that the functor $\operatorname{res}_{\mathcal{D}}^{\mathcal{C}}$ takes (trivial) $\mathcal{D}$-fibrations to (trivial) $\mathcal{D}$-fibrations, so that we have a Quillen adjunction

$$
\operatorname{ind}_{\mathcal{D}}^{\mathcal{C}}: \mathcal{U}_{\mathcal{S}}(\mathcal{D}) \rightleftarrows \mathcal{U}_{\mathcal{S}}(\mathcal{C}, \mathcal{D}): \operatorname{res}_{\mathcal{D}}^{\mathcal{C}} .
$$

The forthcoming two observations will be repeatedly used in the sequel. 
Remark 2.9. For a cofibrantly generated model category $\mathcal{S}$ and two small categories $\mathcal{A}$ and $\mathcal{C}$, the identification $\left(\mathcal{S}^{\mathcal{C}}\right)^{\mathcal{A}}=\mathcal{S}^{\mathcal{C} \times \mathcal{A}}$ of Notation 2.4 is not just an identification of mere categories, but really an identification of model categories (up to the choice of the functorial factorizations):

$$
\mathcal{U}_{\mathcal{U}_{\mathcal{S}}(\mathcal{C})}(\mathcal{A})=\mathcal{U}_{\mathcal{S}}(\mathcal{C} \times \mathcal{A}) \quad \text { or even } \quad \mathcal{U}_{\mathcal{U}_{\mathcal{S}}(\mathcal{C}, \mathcal{D})}(\mathcal{A}, \mathcal{B})=\mathcal{U}_{\mathcal{S}}(\mathcal{C} \times \mathcal{A}, \mathcal{D} \times \mathcal{B})
$$

for any subcategories $\mathcal{D} \subset \mathcal{C}$ and $\mathcal{B} \subset \mathcal{A}$ (the latter $\mathcal{B} \subset \mathcal{A}$ is not used below).

In the next remark, we use the opposite category $\mathcal{A}^{\text {op }}$ of $\mathcal{A}$, instead of $\mathcal{A}$ directly, only for cosmetic reasons justified by the use we make of the remark later on.

Remark 2.10. Let $\mathcal{A}$ and $\mathcal{D} \subset \mathcal{C}$ be small categories and let $\mathcal{S}$ be a cofibrantly generated model category. Suppose that $\mathcal{X}(? ?, ?)$ is a cofibrant diagram in the model category $\mathcal{U}_{\mathcal{S}}\left(\mathcal{A}^{\mathrm{op}} \times \mathcal{C}, \mathcal{A}^{\mathrm{op}} \times \mathcal{D}\right)$. Then, $\mathcal{X}\left(?\right.$ ?, ?) is $\mathcal{A}^{\text {op }}$-objectwise, $\mathcal{C}$-objectwise and $\mathcal{A}^{\text {op }} \times \mathcal{C}$-objectwise cofibrant. More explicitly, for objects $a \in \mathcal{A}$ and $c \in \mathcal{C}$, the three objects $\mathcal{X}(a, ?) \in \mathcal{U}_{\mathcal{S}}(\mathcal{C}, \mathcal{D}), \mathcal{X}(? ?, c) \in \mathcal{U}_{\mathcal{S}}\left(\mathcal{A}^{\text {op }}\right)$ and $\mathcal{X}(a, c) \in \mathcal{S}$ are cofibrant, as follows from Remark 2.7 (cf.Notation 2.4 and Remarks 2.9).

\section{Couplings of categories and couplings of diagrams}

In this section, we consider functors $\odot: \mathcal{S}_{1} \times \mathcal{S}_{2} \longrightarrow \mathcal{S}_{3}$ from a product of two categories to a possibly different category; we call them couplings. This is in particular studied when some of the categories $\mathcal{S}_{i}$ are replaced by the category of $\mathcal{S}_{i}$-valued functors on a given small category. More explicitly, letting $\mathcal{C}$ and $\mathcal{A}$ be small categories, we will induce up two couplings

$$
\stackrel{\mathcal{C}}{\odot}:\left(\mathcal{S}_{1}\right)^{\mathcal{C}} \times \mathcal{S}_{2} \longrightarrow\left(\mathcal{S}_{3}\right)^{\mathcal{C}} \text { and } \underset{\mathcal{A}}{\otimes}:\left(\mathcal{S}_{1}\right)^{\mathcal{A}^{\mathrm{op}}} \times\left(\mathcal{S}_{2}\right)^{\mathcal{A}} \longrightarrow \mathcal{S}_{3} .
$$

Combining both constructions, we will deduce yet another useful coupling

$$
\stackrel{\mathcal{Q}}{\mathcal{A}}_{\mathcal{A}}:\left(\mathcal{S}_{1}\right)^{\mathcal{A}^{\mathrm{op}} \times \mathcal{C}} \times\left(\mathcal{S}_{2}\right)^{\mathcal{A}} \longrightarrow\left(\mathcal{S}_{3}\right)^{\mathcal{C}} \text {. }
$$

The following adjunctions are our basic tools.

Definition 3.1. Let $\mathcal{S}_{1}, \mathcal{S}_{2}$ and $\mathcal{S}_{3}$ be three categories.

(i) An $\mathcal{S}_{3}$-valued coupling of $\mathcal{S}_{1}$ with $\mathcal{S}_{2}$ is a (bi)functor

$$
\odot: \mathcal{S}_{1} \times \mathcal{S}_{2} \longrightarrow \mathcal{S}_{3}, \quad(x, y) \longmapsto x \odot y .
$$

(ii) The coupling $\odot$ is called right tensorial if there exists a functor

$$
\operatorname{map}_{\odot}: \mathcal{S}_{2}^{\text {op }} \times \mathcal{S}_{3} \longrightarrow \mathcal{S}_{1}, \quad(y, z) \longmapsto \operatorname{map}_{\odot}(y, z)
$$

such that for every $x \in \mathcal{S}_{1}, y \in \mathcal{S}_{2}$ and $z \in \mathcal{S}_{3}$, there is a natural bijection

$$
\operatorname{mor}_{\mathcal{S}_{3}}(x \odot y, z) \cong \operatorname{mor}_{\mathcal{S}_{1}}\left(x, \operatorname{map}_{\odot}(y, z)\right) ;
$$

in other words, if for every $y \in \mathcal{S}_{2}$, there is an adjunction

$$
(-) \odot y: \mathcal{S}_{1} \rightleftarrows \mathcal{S}_{3}: \operatorname{map}_{\odot}(y,-)
$$

(necessarily) functorial in $y$; we call map $_{\odot}$ the right mapping functor of $\odot$. 
(iii) The coupling $\odot$ is called left tensorial if there exists a functor

$$
{ }_{\odot} \operatorname{map}: \mathcal{S}_{1}^{\text {op }} \times \mathcal{S}_{3} \longrightarrow \mathcal{S}_{2}, \quad(x, z) \longmapsto{ }_{\odot} \operatorname{map}(x, z)
$$

such that for every $x \in \mathcal{S}_{1}, y \in \mathcal{S}_{2}$ and $z \in \mathcal{S}_{3}$, there is a natural bijection

$$
\operatorname{mor}_{\mathcal{S}_{3}}(x \odot y, z) \cong \operatorname{mor}_{\mathcal{S}_{2}}\left(y,{ }_{\odot} \operatorname{map}(x, z)\right) ;
$$

in other words, if for every $x \in \mathcal{S}_{1}$, there is an adjunction

$$
x \odot(-): \mathcal{S}_{2} \rightleftarrows \mathcal{S}_{3}:{ }_{\odot} \operatorname{map}(x,-)
$$

(necessarily) functorial in $x$; we call $\odot$ map the left mapping functor of $\odot$.

Of course, for a right (resp. left) tensorial coupling the right (resp. left) mapping functor is uniquely determined up to unique natural isomorphism.

Example 3.2. If $\mathcal{S}$ is a category with small coproducts (for example, a cocomplete category), then there is a canonical $\mathcal{S}$-valued coupling of $\mathcal{S}$ ets with $\mathcal{S}$ given by

$$
\square: \operatorname{Sets} \times \mathcal{S} \longrightarrow \mathcal{S}, \quad(K, s) \longmapsto K \boxminus s:=\operatorname{colim}_{K} \underline{s}=\coprod_{K} s,
$$

where in the indicated colimit, we view $K$ as a discrete category, that is, with $K$ as set of objects and only with identity morphisms, and $\underline{s}$ denotes $\operatorname{Cst}_{\mathcal{S}}^{K}(s)$. For $K \in \mathcal{S}$ ets and $s, s^{\prime} \in \mathcal{S}$, the universal property of the coproduct provides a natural bijection

$$
\operatorname{mor}_{\mathcal{S}}\left(\coprod_{K} s, s^{\prime}\right) \cong \operatorname{mor}_{\mathcal{S e t s}}\left(K, \operatorname{mor}_{\mathcal{S}}\left(s, s^{\prime}\right)\right) .
$$

This shows that the coupling $\square$ is right tensorial with right mapping functor mor . $_{\mathcal{S}}$ Note that for the point $* \in \mathcal{S}$ ets and any object $s \in \mathcal{S}$, we have a canonical and natural isomorphism $* \square s \cong s$.

Example 3.3. If $\mathcal{S}$ is a simplicial model category (see Definition A.1), then the "action" $\odot:$ s $\mathcal{S}$ ets $\times \mathcal{S} \longrightarrow \mathcal{S}$ (see Remark A.2) of the category s $\mathcal{S}$ ets of simplicial sets on $\mathcal{S}$ is an $\mathcal{S}$-valued coupling of sSets with $\mathcal{S}$. It is right tensorial with $\operatorname{map}_{\odot}(y, z):=$ $\operatorname{Map}(y, z)$ for every $y, z \in \mathcal{S}$, by virtue of axiom (2) of Definition A.1, using also Definition A.1 (iii). It is left tensorial with $\odot \operatorname{map}(x, z)=z^{x}$ for every $x \in$ sSets and $z \in \mathcal{S}$, by virtue of axiom A.1 (3) of Definition A.1

It turns out that the couplings of Examples 3.2 and 3.3 are "compatible".

Remark 3.4. Let $\mathcal{S}$ be a simplicial model category. Consider the couplings $\square$ of Example 3.2 and $\odot$ of Example 3.3. Then, the following diagram commutes up to natural isomorphism

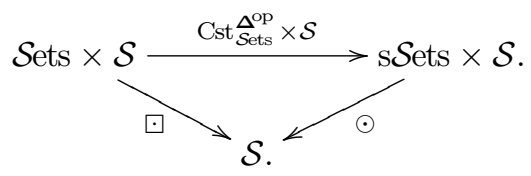

In particular, for the point $*=\Delta^{0} \in$ sSets and an arbitrary object $s \in \mathcal{S}$, there is a natural isomorphism $* \odot s \cong s$. Let us check this. By right adjunction, it suffices 
to prove for all $s \in \mathcal{S}$ the commutativity of

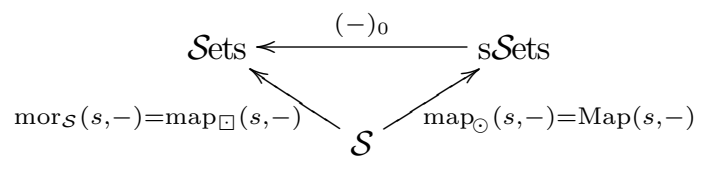

which is precisely one of the axioms of simplicial model categories; see Definition A.1 (iii).

Remark 3.5. If $\odot: \mathcal{S}_{1} \times \mathcal{S}_{2} \longrightarrow \mathcal{S}_{3}$ is a coupling, then the composite functor

$$
{ }^{t} \odot: \mathcal{S}_{2} \times \mathcal{S}_{1} \stackrel{\text { switch }}{\longrightarrow} \mathcal{S}_{1} \times \mathcal{S}_{2} \stackrel{\odot}{\longrightarrow} \mathcal{S}_{3}, \quad y^{t} \odot x:=x \odot y
$$

is also a coupling. It is left (resp. right) tensorial if and only if $\odot$ is right (resp. left) tensorial, with $t_{\odot} \operatorname{map}(?, ? ?):=\operatorname{map}_{\odot}(? ?, ?)$ (and similarly in the other case). Using this observation, we shall focus on right tensorial couplings in the sequel and leave the dual statements to the reader.

We pass to the first construction of induced couplings on categories of diagrams.

Lemma 3.6. Let $\odot: \mathcal{S}_{1} \times \mathcal{S}_{2} \longrightarrow \mathcal{S}_{3}$ be a coupling and let $\mathcal{C}$ be a small category. Then, the assignment $\stackrel{\mathcal{C}}{\mathcal{C}}^{\circ}: \mathcal{S}_{1}^{\mathcal{C}} \times \mathcal{S}_{2}^{\mathcal{C}} \longrightarrow \mathcal{S}_{3}^{\mathcal{C}}, \quad X{ }^{\mathcal{C}} \odot^{\mathcal{C}} Y(?):=X(?) \odot Y(?)$ is a coupling and so is its restriction (called the induced coupling over $\mathcal{C}$ )

$$
\stackrel{\mathcal{c}}{\odot}: \mathcal{S}_{1}^{\mathcal{C}} \times \mathcal{S}_{2} \longrightarrow \mathcal{S}_{3}^{\mathcal{C}}, \quad X \stackrel{\mathcal{C}}{\odot} y(?):=X(?) \odot y
$$

If moreover $\odot$ is right tensorial, then $\stackrel{\mathcal{C}}{\odot}$ is a right tensorial coupling with right mapping functor given by

$$
\operatorname{map}_{\odot}: \mathcal{S}_{2}^{\mathrm{op}} \times \mathcal{S}_{3}^{\mathcal{C}} \longrightarrow \mathcal{S}_{1}^{\mathcal{C}}, \quad \operatorname{map}_{\odot}(y, Z)(?):=\operatorname{map}_{\odot}(y, Z(?)) .
$$

Proof. It is plain that $\stackrel{\mathcal{C}}{\odot}^{\mathcal{c}}$ and $\stackrel{\mathcal{c}}{\odot}$ are couplings and let us prove the moreover part. Let $X \in \mathcal{S}_{1}^{\mathcal{C}}, y \in \mathcal{S}_{2}, Z \in \mathcal{S}_{3}^{\mathcal{C}}$. By adjunction (ii) of Definition 3.1, for a given morphism $\alpha: a \longrightarrow b$ in $\mathcal{C}$, commutative diagrams in $\mathcal{S}_{3}$ like $(*)$
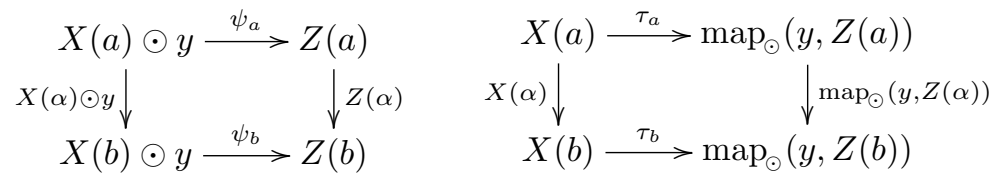

are in one-one correspondence with commutative diagrams in $\mathcal{S}_{1}$ like $(* *)$. The set

$$
\left\{\left(\psi_{c}\right)_{c \in \mathcal{C}} \in \prod_{c \in \mathcal{C}} \operatorname{mor}_{\mathcal{S}_{3}}(X(c) \odot y, Z(c)) \mid(*) \text { commutes } \forall \alpha: a \rightarrow b \text { in } \mathcal{C}\right\},
$$

which coincides with the set $\operatorname{mor}_{\mathcal{S}_{3}^{\mathcal{C}}}(X \stackrel{\mathcal{C}}{\odot} y, Z)$, is thus in bijection with the set

$$
\left\{\left(\tau_{c}\right)_{c \in \mathcal{C}} \in \prod_{c \in \mathcal{C}} \operatorname{mor}_{\mathcal{S}_{3}}\left(X(c), \operatorname{map}_{\odot}(y, Z(c))\right) \mid(* *) \text { commutes } \forall \alpha: a \rightarrow b \text { in } \mathcal{C}\right\}
$$

which is equal to $\operatorname{mor}_{\mathcal{S}_{1}^{c}}\left(X, \operatorname{map}_{\odot}(y, Z)\right)$. The rest is routine. 
Remark 3.7. With notation as in Lemma 3.6, we warn the hurried reader that given $Y \in \mathcal{S}_{2}^{\mathcal{C}}$ and $Z \in \mathcal{S}_{3}^{\mathcal{C}}$, there is no reasonable way of making a functor out of the objectwise assignment $c \mapsto \operatorname{map}_{\odot}(Y(c), Z(c))$.

Notation 3.8. Let $\odot: \mathcal{S}_{1} \times \mathcal{S}_{2} \longrightarrow \mathcal{S}_{3}$ be a coupling and $\mathcal{C}$ a small category. The coupling $\stackrel{\mathcal{C}}{\odot}$ of Lemma 3.6 has a second possible restriction, denoted by

$$
\odot^{\mathcal{C}}: \mathcal{S}_{1} \times \mathcal{S}_{2}^{\mathcal{C}} \longrightarrow \mathcal{S}_{3}^{\mathcal{C}}, \quad\left(x \odot^{\mathcal{C}} Y\right)(?):=x \odot(Y(?)) .
$$

Next, we study a second way to induce up couplings on categories of diagrams.

Recall from [13, pp. 64-65] the notion of co-equalizer of a pair $s_{1} \stackrel{f}{\underset{g}{\longrightarrow}} s_{2}$ of morphisms in a category $\mathcal{S}$, with same source and same target: it is defined by

$$
\operatorname{coeq}\left(s_{1} \underset{g}{\stackrel{f}{\longrightarrow}} s_{2}\right):=\underset{\bullet \rightarrow \bullet}{\operatorname{colim}}\left(s_{1} \underset{g}{\stackrel{f}{\longrightarrow}} s_{2}\right),
$$

the universal morphism (or only its target) out of $s_{2}$ which "co-equalizes" $f$ and $g$.

Definition 3.9. Let $\odot: \mathcal{S}_{1} \times \mathcal{S}_{2} \longrightarrow \mathcal{S}_{3}$ be a coupling, with $\mathcal{S}_{3}$ cocomplete, and let $\mathcal{A}$ be a small category. The tensor product over $\mathcal{A}$ associated to $\odot$ is the assignment

$$
\otimes_{\mathcal{A}}: \mathcal{S}_{1}^{\mathcal{A}^{\mathrm{op}}} \times \mathcal{S}_{2}^{\mathcal{A}} \longrightarrow \mathcal{S}_{3}, \quad(X, Y) \longmapsto X \otimes_{\mathcal{A}} Y
$$

defined for $X \in \mathcal{S}_{1}^{\mathcal{A}^{\mathrm{op}}}$ and $Y \in \mathcal{S}_{2}^{\mathcal{A}}$ by the formula

$$
X \otimes_{\mathcal{A}} Y=\operatorname{coeq}\left(\coprod_{\left(b \stackrel{\alpha}{\rightarrow} b^{\prime}\right) \in \mathcal{A}} X\left(b^{\prime}\right) \odot Y(b) \Longrightarrow \coprod_{a \in \mathcal{A}} X(a) \odot Y(a)\right)
$$

where the two indicated morphisms inside the co-equalizer are given, on a summand indexed by $\alpha: b \longrightarrow b^{\prime}$, by the compositions

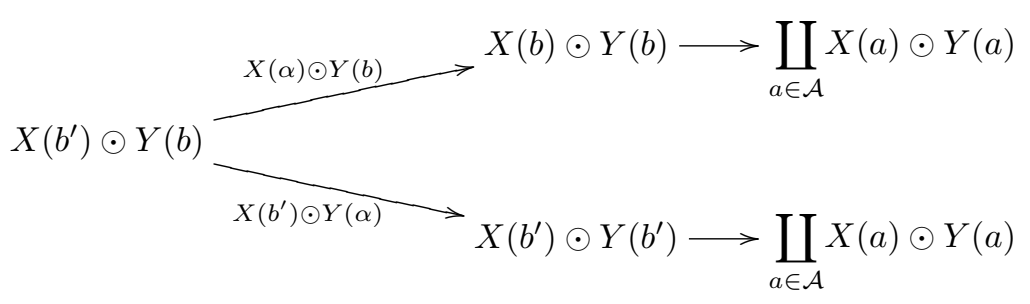

It is sometimes useful to write $X \underset{\mathcal{A}}{\otimes} Y$ as $X(?) \otimes_{\mathcal{A}} Y(?)$ or even as $X(?) \underset{? \in \mathcal{A}}{\otimes} Y(?)$.

Example 3.10. Let $\odot: \mathcal{S}_{1} \times \mathcal{S}_{2} \longrightarrow \mathcal{S}_{2}$ be a coupling (note that $\mathcal{S}_{3}=\mathcal{S}_{2}$ ). That is, $\odot$ is an "action" of a category $\mathcal{S}_{1}$ on $\mathcal{S}_{2}$. Suppose that $\mathcal{S}_{2}$ is cocomplete. Assume that there exists an object $x_{0} \in \mathcal{S}_{1}$ such that the "action" of $x_{0}$ is trivial, more precisely, such that the functor $x_{0} \odot(-)$ is isomorphic to the identity functor of $\mathcal{S}_{2}$. Then, 
for every small category $\mathcal{A}$, there is an isomorphism of functors

$$
\underline{x_{0}} \underset{\mathcal{A}}{\otimes}(-) \cong \operatorname{colim}_{\mathcal{A}}(-): \quad \mathcal{S}_{2}^{\mathcal{A}} \longrightarrow \mathcal{S}_{2}
$$

where $\underline{x_{0}} \in \mathcal{S}_{1}^{\mathcal{A}^{\text {op }}}$ is the constant diagram with value $x_{0} \in \mathcal{S}_{1}$. Indeed, for $Y \in \mathcal{S}_{2}^{\mathcal{A}}$, one obtains a natural isomorphism

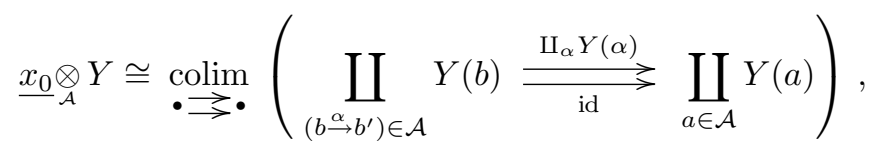

and the latter is easily seen to satisfy the universal property of the colimit of $Y$ over $\mathcal{A}$. A typical situation where this applies is for $\odot$ as in Example 3.3, with $\mathcal{S}_{1}:=$ s Sets, $\mathcal{S}_{2}:=\mathcal{S}$ (a simplicial model category) and with $x_{0}:=\Delta^{0} \in$ sets.

Lemma 3.11. Let $\odot: \mathcal{S}_{1} \times \mathcal{S}_{2} \longrightarrow \mathcal{S}_{3}$ be a coupling, with $\mathcal{S}_{3}$ cocomplete, and let $\mathcal{A}$ be a small category. Suppose that $\odot$ is right tensorial. Then, the assignment

$$
\otimes_{\mathcal{A}}: \mathcal{S}_{1}^{\mathcal{A}^{\mathrm{op}}} \times \mathcal{S}_{2}^{\mathcal{A}} \longrightarrow \mathcal{S}_{3}
$$

is a right tensorial coupling with right mapping functor

$$
\operatorname{map}_{\underset{\mathcal{A}}{\otimes}}:\left(\mathcal{S}_{2}^{\mathcal{A}}\right)^{\mathrm{op}} \times \mathcal{S}_{3} \longrightarrow \mathcal{S}_{1}^{\mathcal{A}^{\mathrm{op}}}, \quad \operatorname{map}_{\otimes_{\mathcal{A}}}(Y, z)(?):=\operatorname{map}_{\odot}(Y(?), z) .
$$

Proof. The fact that $\otimes_{\mathcal{A}}$ is a functor is a straightforward checking. Let $X \in \mathcal{S}_{1}^{\mathcal{A}^{\text {op }}}$, $Y \in \mathcal{S}_{2}^{\mathcal{A}}$ and $z \in \mathcal{S}_{3}$. By definition of co-equalizers, the set $\operatorname{mor}_{\mathcal{S}_{3}}(X \otimes Y, z)$ is equal to the set of those elements $\left(\psi_{a}\right)_{a \in \mathcal{A}}$ in $\prod_{a \in \mathcal{A}} \operatorname{mor}_{\mathcal{S}_{3}}(X(a) \odot Y(a), z)$ such that for every morphism $\alpha: b \longrightarrow b^{\prime}$ in $\mathcal{A}$, the following left-hand (or equivalently right-hand) diagram commutes:
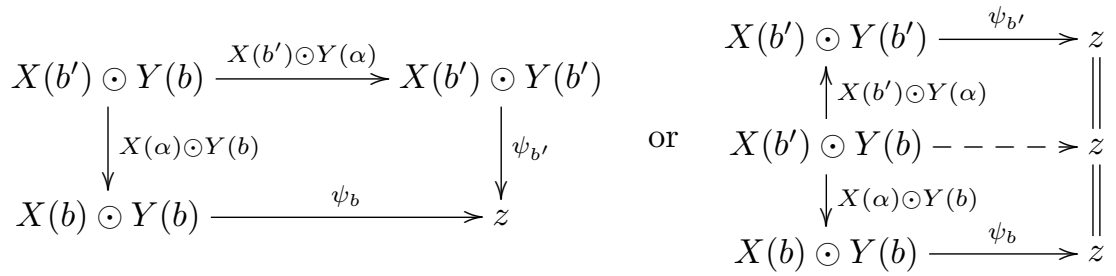

By adjunction (ii) of Definition 3.1, this set is in bijection with the set of those tuples $\left(\tau_{a}\right)_{a \in \mathcal{A}}$ in $\prod_{a \in \mathcal{A}}$ mor $_{\mathcal{S}_{1}}\left(X(a), \operatorname{map}_{\odot}(Y(a), z)\right)$ making the following left-hand (or equivalently right-hand) diagram commutative for every morphism $\alpha: b \longrightarrow b^{\prime}$ in $\mathcal{A}$ :

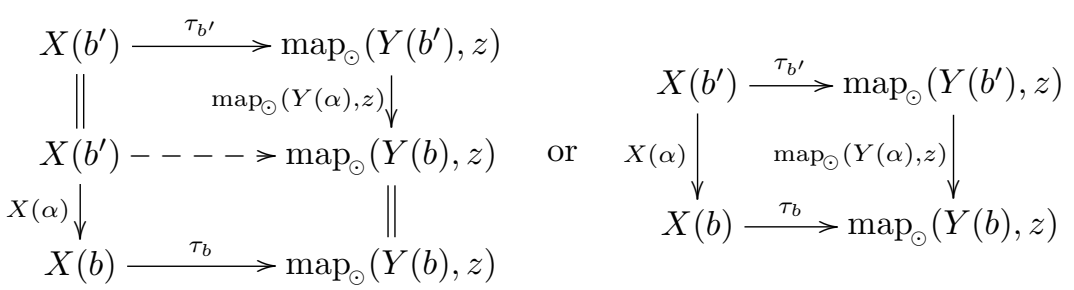


This set is nothing but $\operatorname{mor}_{\mathcal{S}^{\mathcal{A}} \text { op }}\left(X(?), \operatorname{map}_{\odot}(Y(?), z)\right)$, as was to be shown, the required naturality properties being, again, routine.

Mixing the two ways of inducing couplings seen so far, we have the immediate:

Proposition-Definition 3.12. Let $\odot: \mathcal{S}_{1} \times \mathcal{S}_{2} \longrightarrow \mathcal{S}_{3}$ be a coupling, with $\mathcal{S}_{3}$ cocomplete. Consider two small categories $\mathcal{A}$ and $\mathcal{C}$. Applying successively the constructions of Lemmas 3.11 and 3.6, we obtain a coupling

$$
{ }^{\mathcal{C}}\left({ }_{\mathcal{A}}^{\otimes}\right): \mathcal{S}_{1}^{\mathcal{A}^{\mathrm{op}} \times \mathcal{C}} \times \mathcal{S}_{2}^{\mathcal{A}} \longrightarrow \mathcal{S}_{3}^{\mathcal{C}}
$$

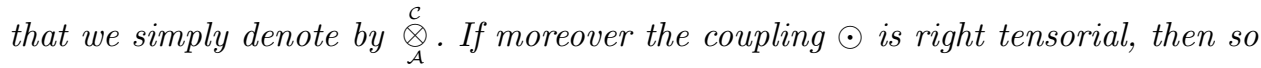
$i s \stackrel{\mathcal{A}}{\mathcal{A}}^{\mathcal{C}}$ and its right mapping functor reads

$$
\operatorname{map}_{\substack{\mathscr{\mathcal { A }} \\ \mathcal{\mathcal { A }}}}:\left(\mathcal{S}_{2}^{\mathcal{A}}\right)^{\mathrm{op}} \times \mathcal{S}_{3}^{\mathcal{C}} \longrightarrow \mathcal{S}_{1}^{\mathcal{A}^{\mathrm{op}} \times \mathcal{C}}, \quad \operatorname{map}_{\substack{\mathcal{A} \\ \mathcal{A}}}(Y, Z)(? ?, ?):=\operatorname{map}_{\odot}(Y(? ?), Z(?))
$$

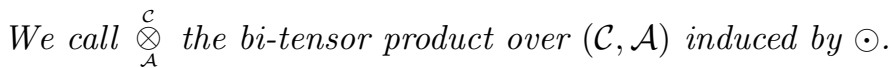

Explicitly, for $\mathcal{X} \in \mathcal{S}_{1}^{\mathcal{A}^{\mathrm{op}} \times \mathcal{C}}$, for $Y \in \mathcal{S}_{2}^{\mathcal{A}}$ and for $c \in \mathcal{C}$, we have

$$
\begin{aligned}
\mathcal{X} \stackrel{\mathcal{A}}{\otimes}_{\mathcal{A}} Y(c) & =\mathcal{X}(? ?, c){\underset{? ? \in \mathcal{A}}{\otimes}} Y(? ?) \\
& =\operatorname{coeq}\left(\coprod_{\left(b \stackrel{\alpha}{\rightarrow} b^{\prime}\right) \in \mathcal{A}} \mathcal{X}\left(b^{\prime}, c\right) \odot Y(b) \longrightarrow \coprod_{a \in \mathcal{A}} \mathcal{X}(a, c) \odot Y(a)\right) .
\end{aligned}
$$

We often designate $\mathcal{X}(? ?, ?) \stackrel{\mathcal{C}}{\otimes} Y(? ?)$ simply by $\mathcal{X} \stackrel{\mathcal{A}}{\mathcal{A}}_{\text {? }} Y$; this should cause no confusion: the tensor product is performed over the contravariant variable of $\mathcal{X}$. The coupling obtained in the other order, i.e. first Lemma 3.6 and then Lemma 3.11, is isomorphic to the above one up to precomposition with the obvious switch functor $\sigma_{\mathcal{C}, \mathcal{A}}$ op $\times \mathcal{S}_{2}^{\mathcal{A}}$ (see Notation 2.4). Indeed, unfolding the definitions gives back the same formula.

Example 3.13. Let $\mathcal{S}$ be a cofibrantly generated simplicial model category, and let $\bullet$ and $\odot$ be the couplings of Examples 3.2 and 3.3. Given two small categories $\mathcal{A}$ and $\mathcal{C}$, we get two bi-tensor products

$$
{\underset{\mathcal{A}}{\mathcal{C}}}^{\mathcal{C}}: \operatorname{Sets}^{\mathcal{A}^{\mathrm{op}} \times \mathcal{C}} \times \mathcal{S}^{\mathcal{A}} \longrightarrow \mathcal{S}^{\mathcal{C}} \text { and } \quad \stackrel{\mathcal{Q}}{\mathcal{A}}^{\mathcal{C}}: \operatorname{sets}^{\mathcal{A}^{\mathrm{op}}} \times \mathcal{C} \times \mathcal{S}^{\mathcal{A}} \longrightarrow \mathcal{S}^{\mathcal{C}}
$$

Given $Y \in \mathcal{S}^{\mathcal{A}}$ and $Z \in \mathcal{S}^{\mathcal{C}}$, we get for $\mathcal{X} \in \mathcal{S e t s}^{\mathcal{A}^{\text {op }} \times \mathcal{C}}$, a natural bijection

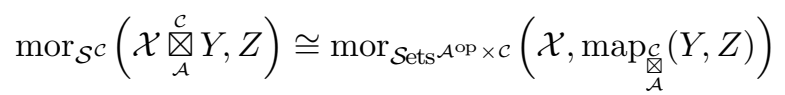

and for $\mathcal{X}^{\prime} \in \operatorname{sSets}^{\mathcal{A}^{\mathrm{op}} \times \mathcal{C}}$, a natural bijection

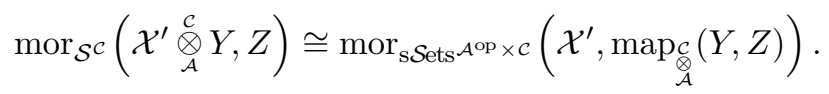

Furthermore, the right mapping functors are explicitly given by

$$
\operatorname{map}_{\substack{\mathscr{\mathcal { M }} \\ \mathcal{M}}}(Y, Z)(? ?, ?)=\operatorname{mor}_{\mathcal{S}}(Y(? ?), Z(?))
$$


and

$$
\operatorname{map}_{\mathcal{A}}(Y, Z)(? ?, ?)=\operatorname{Map}(Y(? ?), Z(?))
$$

Remark 3.14. Keep notations as in Proposition-Definition 3.12. Let $\mathcal{D}$ be a subcategory of $\mathcal{C}$ and let

$$
\operatorname{res}_{\mathcal{A}^{\mathrm{op}} \times \mathcal{D}}^{\mathcal{o p}_{\mathcal{D}}}: \mathcal{S}_{1}^{\mathcal{A}^{\mathrm{op}} \times \mathcal{C}} \longrightarrow \mathcal{S}_{1}^{\mathcal{A}^{\mathrm{op}} \times \mathcal{D}} \text { and } \quad \operatorname{res}_{\mathcal{D}}^{\mathcal{C}}: \mathcal{S}_{3}^{\mathcal{C}} \longrightarrow \mathcal{S}_{3}^{\mathcal{D}}
$$

be the obvious restriction functors. Then, for $\mathcal{X} \in \mathcal{S}_{1}^{\mathcal{A}^{\mathrm{op}} \times \mathcal{C}}$ and $Y \in \mathcal{S}_{2}^{\mathcal{A}}$, one has

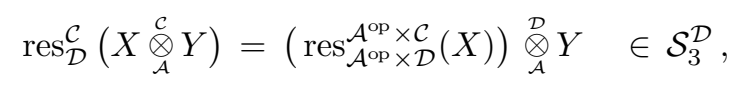

as evaluation at an arbitrary object $d \in \mathcal{D}$ immediately shows.

\section{Couplings and model category structures}

We study when the functor obtained from a coupling of model categories by fixing the second variable preserves some weak equivalences. For this purpose, the next definition turns crucial. The origin of such a concept goes back to Kan's homotopy lifting extension theorem for categories of simplicial objects (in some category) enriched over sSets, see [11]. This was then taken as axiom (SM7) for a simplicial model category by Quillen in [14] (see axiom (4) in Definition A.1).

Definition 4.1. Let $\mathcal{S}_{1}, \mathcal{S}_{2}$ and $\mathcal{S}_{3}$ be model categories. Let $\odot: \mathcal{S}_{1} \times \mathcal{S}_{2} \longrightarrow \mathcal{S}_{3}$ be a right tensorial coupling, with right mapping functor map. ${ }_{\odot}$. We say that $\odot$ has the corner-map property if the following holds: for every cofibration $i: y \longrightarrow y^{\prime}$ in $\mathcal{S}_{2}$ and for every fibration $p: z \longrightarrow z^{\prime}$ in $\mathcal{S}_{3}$, the induced morphism in $\mathcal{S}_{1}$

$$
\varphi: \operatorname{map}_{\odot}\left(y^{\prime}, z\right) \longrightarrow \operatorname{map}_{\odot}(y, z) \times_{\operatorname{map}_{\odot}\left(y, z^{\prime}\right)} \operatorname{map}_{\odot}\left(y^{\prime}, z^{\prime}\right)
$$

is a fibration, and it is a trivial fibration if either $i$ or $p$ is moreover a weak equivalence. This morphism $\varphi$ is the "corner map" to the pull-back induced by the morphisms $\operatorname{map}_{\odot}(i, z)$ and $\operatorname{map}_{\odot}\left(y^{\prime}, p\right)$ as follows:

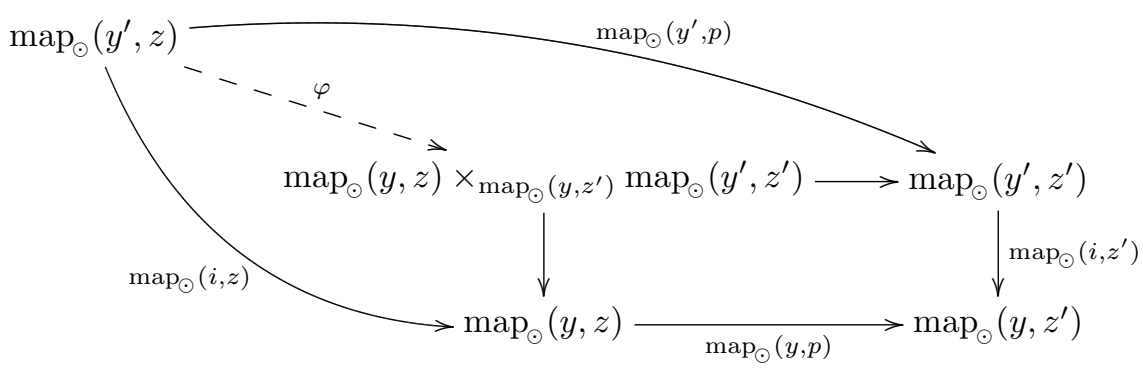


Example 4.2. For a simplicial model category $\mathcal{S}$, the right tensorial coupling $\odot$ given by the "action" of the category sSets on $\mathcal{S}$, see Example 3.3, has the corner-map property, by Axiom (4) of Definition A.1.

Remark 4.3. Let $\odot: \mathcal{S}_{1} \times \mathcal{S}_{2} \longrightarrow \mathcal{S}_{3}$ be a right tensorial coupling having the cornermap property. Let $y \in \mathcal{S}_{2}$ be a cofibrant object. Applying the above condition to the cofibration $i: \varnothing \longrightarrow y$, it is easy to check that the functor $\operatorname{map}_{\odot}(y,-): \mathcal{S}_{3} \longrightarrow \mathcal{S}_{1}$ preserves (trivial) fibrations. In other words, we have a Quillen adjunction

$$
-\odot y: \mathcal{S}_{1} \rightleftarrows \mathcal{S}_{3}: \operatorname{map}_{\odot}(y,-)
$$

In particular, the functor $-\odot y$ preserves (trivial) cofibrations. So, $-\odot y$ preserves cofibrant objects and weak-equivalences between them, by Ken Brown's lemma [10, Lemma 1.1.12].

Of course, we want to extend the above corner-map property to categories of diagrams. The obvious statement holds (Corollary 4.5) but is not sufficient for applications. We shall need the following improved version. Note that the morphism $i$ of the statement is only required to be an objectwise cofibration.

Lemma 4.4. Let $\mathcal{S}_{1}, \mathcal{S}_{2}$ and $\mathcal{S}_{3}$ be cofibrantly generated model categories. Let $\odot: \mathcal{S}_{1} \times \mathcal{S}_{2} \longrightarrow \mathcal{S}_{3}$ be a right tensorial coupling with the corner-map property. Let $\mathcal{A}$ and $\mathcal{C}$ be small categories and let $\mathcal{D} \subset \mathcal{C}$ be a subcategory. Consider

$$
\operatorname{map}_{\mathcal{A}}:\left(\mathcal{S}_{2}^{\mathcal{A}}\right)^{\mathrm{op}} \times \mathcal{S}_{3}^{\mathcal{C}} \longrightarrow \mathcal{S}_{1}^{\mathcal{A}^{\mathrm{op}} \times \mathcal{C}}
$$

the right mapping functor of the bi-tensor product $\stackrel{\bigotimes}{\mathcal{A}}_{\mathcal{C}}: \mathcal{S}_{1}^{\mathcal{A}^{\mathrm{op}} \times \mathcal{C}} \times \mathcal{S}_{2}^{\mathcal{A}} \longrightarrow \mathcal{S}_{3}^{\mathcal{C}}$ from Proposition-Definition 3.12. Assume that $i: Y \rightarrow Y^{\prime}$ is an objectwise cofibration in $\mathcal{S}_{2}^{\mathcal{A}}$ and that $p: Z \rightarrow Z^{\prime}$ in $\mathcal{S}_{3}^{\mathcal{C}}$ is a (trivial) fibration in $\mathcal{U}_{\mathcal{S}_{3}}(\mathcal{C}, \mathcal{D})$. Then the "cornermap" morphism

$$
\Phi: \operatorname{map}_{\substack{\mathcal{Q} \\ \mathcal{A}}}\left(Y^{\prime}, Z\right) \longrightarrow \operatorname{map}_{\substack{\mathcal{Q} \\ \mathcal{A}}}(Y, Z) \underset{\operatorname{map}_{\substack{\mathcal{Q} \\ \mathcal{A}}}^{\times}\left(Y, Z^{\prime}\right)}{\times} \operatorname{map}_{\substack{\mathcal{Q} \\ \mathcal{A}}}\left(Y^{\prime}, Z^{\prime}\right)
$$

is a (trivial) fibration in $\mathcal{U}_{\mathcal{S}_{1}}\left(\mathcal{A}^{\mathrm{op}} \times \mathcal{C}, \mathcal{A}^{\mathrm{op}} \times \mathcal{D}\right)$.

Proof. Recall from Proposition 3.12, where we saw that $\stackrel{\mathcal{A}}{\mathcal{A}}_{\text {is }}$ ight tensorial, that

$$
\operatorname{map}_{\substack{\mathcal{A} \\ \mathcal{A}}}(Y, Z)(? ?, ?):=\operatorname{map}_{\odot}(Y(? ?), Z(?))
$$

Since the pull-back and the morphism $\Phi$ are defined in the diagram-category $\mathcal{S}_{1}^{\mathcal{A}^{\mathrm{op}} \times \mathcal{C}}$, they can be computed $\mathcal{A}^{\text {op }} \times \mathcal{C}$-objectwise. Let $a$ be an object of $\mathcal{A}$ and let $d$ be an 
object of $\mathcal{D} \subset \mathcal{C}$. The "corner map" $\Phi(a, d)$ is the following map:

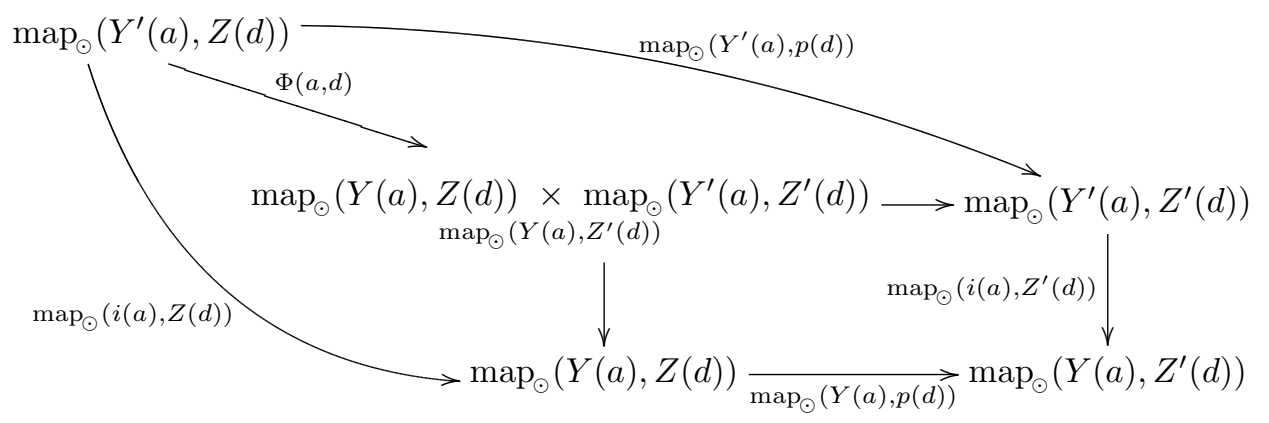

By assumption, $i(a): Y(a) \longrightarrow Y^{\prime}(a)$ is a cofibration in $\mathcal{S}_{2}$ and $p(d): Z(d) \longrightarrow Z^{\prime}(d)$ is a (trivial) fibration in $\mathcal{S}_{3}$. So, $\Phi(a, d)$ indeed is a (trivial) fibration since the original coupling $\odot: \mathcal{S}_{1} \times \mathcal{S}_{2} \longrightarrow \mathcal{S}_{3}$ has the corner map property.

Corollary 4.5. Let $\mathcal{S}_{1}, \mathcal{S}_{2}$ and $\mathcal{S}_{3}$ be cofibrantly generated model categories. Let $\odot: \mathcal{S}_{1} \times \mathcal{S}_{2} \longrightarrow \mathcal{S}_{3}$ be a right tensorial coupling with the corner-map property. Let $\mathcal{A}$ and $\mathcal{C}$ be small categories and let $\mathcal{D} \subset \mathcal{C}$ be a subcategory. Then the bi-tensor product $\stackrel{\mathcal{A}}{\mathcal{C}}_{\mathcal{C}}$ has the corner map property for the following model structures:

$$
\stackrel{\mathcal{C}}{\otimes}: \mathcal{U}_{\mathcal{S}_{1}}\left(\mathcal{A}^{\mathrm{op}} \times \mathcal{C}, \mathcal{A}^{\mathrm{op}} \times \mathcal{D}\right) \times \mathcal{U}_{\mathcal{S}_{2}}(\mathcal{A}) \longrightarrow \mathcal{U}_{\mathcal{S}_{3}}(\mathcal{C}, \mathcal{D})
$$

Proof. This is a direct consequence of Lemma 4.4 since a cofibration $i: Y \rightarrow Y^{\prime}$ in $\mathcal{U}_{\mathcal{S}_{2}}(\mathcal{A})$ is in particular $\mathcal{A}$-objectwise a cofibration (see Remark 2.7).

Our next goal is to single out some situations where taking the coupling with a given object preserves weak equivalence.

Theorem 4.6. Let $\mathcal{S}_{1}, \mathcal{S}_{2}$ and $\mathcal{S}_{3}$ be cofibrantly generated model categories and let $\odot: \mathcal{S}_{1} \times \mathcal{S}_{2} \longrightarrow \mathcal{S}_{3}$ be a right tensorial coupling having the corner-map property. Let $\mathcal{A}$ and $\mathcal{C}$ be small categories and $\mathcal{D}$ a subcategory of $\mathcal{C}$. Let $Y \in \mathcal{S}_{2}^{\mathcal{A}}$ be $\mathcal{A}$-objectwise cofibrant. Then, there is a Quillen adjunction

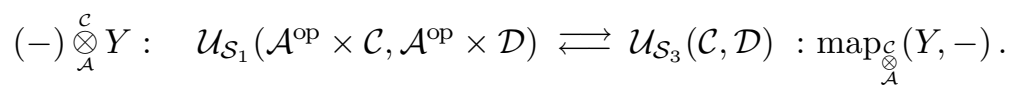

In particular, in this situation, the functor $(-) \stackrel{\mathcal{Q}}{\mathcal{A}}^{\mathcal{C}} Y$ takes $\mathcal{A}^{\mathrm{op}} \times \mathcal{D}$-weak equivalences between cofibrant objects in $\mathcal{U}_{\mathcal{S}_{1}}\left(\mathcal{A}^{\mathrm{op}} \times \mathcal{C}, \mathcal{A}^{\mathrm{op}} \times \mathcal{D}\right)$ to $\mathcal{D}$-weak equivalences between cofibrant objects in $\mathcal{U}_{\mathcal{S}_{3}}(\mathcal{C}, \mathcal{D})$.

Proof. It suffices to show that $\operatorname{map}_{\substack{\mathcal{A} \\ \mathcal{A}}}(Y,-): \mathcal{U}_{\mathcal{S}_{3}}(\mathcal{C}, \mathcal{D}) \longrightarrow \mathcal{U}_{\mathcal{S}_{1}}\left(\mathcal{A}^{\text {op }} \times \mathcal{C}, \mathcal{A}^{\text {op }} \times \mathcal{D}\right)$ preserves fibrations and trivial fibrations, which are tested objectwise in both the source and target model categories. Let $p: Z \longrightarrow Z^{\prime}$ be a (trivial) $\mathcal{D}$-fibration in $\mathcal{S}_{3}^{\mathcal{C}}$. We conclude by Lemma 4.4 applied to $i: \varnothing \longrightarrow Y$ and to our $p: Z \longrightarrow Z^{\prime}$. 
We leave it to the reader to unfold the obvious corollaries of the results of the present section when $\mathcal{A}$ is reduced to a point or when $\mathcal{D}=\mathcal{C}$. We only mention for further quotation the following consequence of Theorem 4.6 with $\mathcal{D}=\mathcal{C}=\{*\}$.

Corollary 4.7. Let $\mathcal{S}_{1}, \mathcal{S}_{2}$ and $\mathcal{S}_{3}$ be cofibrantly generated model categories and let $\odot: \mathcal{S}_{1} \times \mathcal{S}_{2} \longrightarrow \mathcal{S}_{3}$ be a right tensorial coupling having the corner-map property. Let $\mathcal{A}$ be a small category. Let $Y \in \mathcal{S}_{2}^{\mathcal{A}}$ be $\mathcal{A}$-objectwise cofibrant. Let $\xi: X \longrightarrow$ $X^{\prime}$ in $\mathcal{S}_{1}^{\mathcal{A}^{\mathrm{op}}}$ be a weak equivalence between cofibrant objects in $\mathcal{U}_{\mathcal{S}_{1}}\left(\mathcal{A}^{\mathrm{op}}\right)$. Then the morphism $\otimes_{\mathcal{A}} Y: X \otimes_{\mathcal{A}} Y \longrightarrow X^{\prime} \otimes_{\mathcal{A}} Y$ is a weak equivalence in $\mathcal{S}_{3}$.

\section{Cofibrant approximations in $\mathcal{U}_{\mathcal{S}}(\mathcal{C}, \mathcal{D})$}

The goal in this section is to establish a general technique to construct functorial cofibrant approximation in the model category $\mathcal{U}_{\mathcal{S}}(\mathcal{C}, \mathcal{D})$. Explicit examples will be presented in Section 6. The reader who prefers to focus on the model category $\mathcal{U}_{\mathcal{S}}(\mathcal{C})$ may well take $\mathcal{D}=\mathcal{C}$ everywhere in the present section, although in this case, some proofs look more transparent when distinguishing two different occurrences of $\mathcal{C}$, calling one of them $\mathcal{D}$ and the other one $\mathcal{C}$ as we do here.

Notation 5.1. Let $\mathcal{C}$ be a small category. We fix the following notations for the rest of the paper.

(i) Let $\mathcal{S}$ be a model category with cofibrant replacement denoted by $\left(Q_{\mathcal{S}}, \xi^{\mathcal{S}}\right)$. For a diagram $X \in \mathcal{S}^{\mathcal{C}}$, we denote by $q X$ the diagram $Q_{\mathcal{S}} \circ X \in \mathcal{S}^{\mathcal{C}}$ obtained by applying the cofibrant replacement $\mathcal{C}$-objectwise to $X$, that is,

$$
q X: \mathcal{C} \stackrel{X}{\longrightarrow} \mathcal{S} \stackrel{Q_{\mathcal{S}}}{\longrightarrow} \mathcal{S}, \quad q X(c)=Q_{\mathcal{S}}(X(c)) .
$$

We let $\eta_{X}: q X \longrightarrow X$ be the morphism given, for $c \in \mathcal{C}$, by $\eta_{X}(c):=\xi_{X(c)}^{\mathcal{S}}$.

(ii) For a subcategory $\mathcal{D}$ of $\mathcal{C}$, we define the diagram of sets

$$
\operatorname{mor}_{\mathcal{D}, \mathcal{C}}: \mathcal{D}^{\text {op }} \times \mathcal{C} \longrightarrow \mathcal{S e t s}, \quad(d, c) \longmapsto \operatorname{mor}_{\mathcal{C}}(d, c) .
$$

Composing with the usual constantification functor $\mathrm{Cst}_{\mathcal{S} \text { ets }}^{\Delta^{\text {op }}}: \mathcal{S}$ ets $\longrightarrow \mathrm{s} \mathcal{S}$ ets, we obtain the functor $\mathbb{F}_{\mathcal{D}}^{\mathcal{C}}:=$ mor $_{\mathcal{D}, \mathcal{C}}$, that is,

$$
\mathbb{F}_{\mathcal{D}}^{\mathcal{C}}: \mathcal{D}^{\mathrm{op}} \times \mathcal{C} \longrightarrow \mathrm{s} \mathcal{S e t s}, \quad(d, c) \longmapsto \operatorname{mor}_{\mathcal{C}}(d, c) .
$$

When $\mathcal{D}=\mathcal{C}$, we shall abbreviate $\mathbb{F}_{\mathcal{C}}^{\mathcal{C}}$ by $\mathbb{F} \mathcal{C}$ but we still write mor $_{\mathcal{C}, \mathcal{C}}$ to stress the difference between this diagram of sets and the mere set mor $_{\mathcal{C}}$.

Here is the first result regarding the diagram $\mathbb{F}_{\mathcal{D}}^{\mathcal{C}}$, giving it some flavour. 
Lemma 5.2. Let $\mathcal{D}$ be a full subcategory of a small category $\mathcal{C}$. Then, there are canonical isomorphisms

$$
\operatorname{ind}_{\mathcal{D}^{\mathrm{op}} \times \mathcal{D}}^{\mathcal{D}_{\mathrm{op}}}\left(\operatorname{mor}_{\mathcal{D}, \mathcal{D}}\right) \cong \operatorname{mor}_{\mathcal{D}, \mathcal{C}} \in \mathcal{S e t s}^{\mathcal{D}^{\mathrm{op}} \times \mathcal{C}}
$$

and

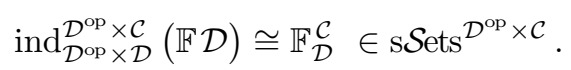

¿From now on, for simplicity, we consider these identifications as equalities.

Proof. For an object $d \in \mathcal{D}^{\text {op }}$, it suffices to produce in $\mathcal{S e t s}^{\mathcal{C}}$ an isomorphism $\operatorname{ind}_{\mathcal{D}}^{\mathcal{C}}\left(\operatorname{mor}_{\mathcal{D}}(d, ?)\right) \longrightarrow \operatorname{mor}_{\mathcal{D}, \mathcal{C}}(d, ?)=\operatorname{mor}_{\mathcal{C}}(d, ?)$ that is natural in $d$. The induction functor being a left Kan extension (see [13] or [1, Definition B.2]), we have for all $c \in \mathcal{C}$

$$
\left(\operatorname{ind}_{\mathcal{D}}^{\mathcal{C}}\left(\operatorname{mor}_{\mathcal{D}}(d, ?)\right)\right)(c) \cong \underset{\mathcal{D} \backslash c}{\operatorname{colim}} \operatorname{mor}_{\mathcal{D}}\left(d, d^{\prime}\right)=\underset{\mathcal{D} \backslash c}{\operatorname{colim}_{\searrow}} \operatorname{mor}_{\mathcal{C}}\left(d, d^{\prime}\right) \cong \operatorname{mor}_{\mathcal{C}}(d, c),
$$

where the colimits are taken over $\left(d^{\prime}, d^{\prime} \stackrel{\alpha}{\rightarrow} c\right) \in \mathcal{D} \backslash c$. The equality in the middle follows from fullness. The last isomorphism is easy. The second part follows.

Lemma 5.3. Let $\mathcal{S}$ be a simplicial model category and $\mathcal{D}$ a full subcategory of a small category $\mathcal{C}$. Let $\underset{\mathcal{D}}{\stackrel{\mathcal{D}}{\otimes}}$ and $\underset{\mathcal{D}}{\stackrel{\mathcal{D}}{D}}$ be the bi-tensor products induced by the couplings $\square$ of 3.2 and $\odot$ of 3.3, respectively. Then, for every diagram $X \in \mathcal{S}^{\mathcal{D}}$, there are canonical and natural isomorphisms

$$
\lambda_{X}^{\mathcal{D}, \mathcal{C}}: \quad \mathbb{F}_{\mathcal{D}}^{\mathcal{C}}(? ?, ?) \stackrel{\mathcal{C}}{\underset{\mathcal{D}}{\otimes}} X(? ?) \stackrel{\cong}{\longrightarrow} \operatorname{mor}_{\mathcal{D}, \mathcal{C}}(? ?, ?) \stackrel{\stackrel{\mathcal{X}}{\mathbb{D}}}{ } X(? ?) \stackrel{\cong}{\longrightarrow} \operatorname{ind}_{\mathcal{D}}^{\mathcal{C}} X(?) .
$$

Proof. The first isomorphism follows from Remark 3.4. So, it suffices to construct the second isomorphism, for which it is enough to check that the functor $\operatorname{mor}_{\mathcal{D}, \mathcal{C}} \underset{\mathcal{D}}{\stackrel{\mathcal{D}}{\mathbb{D}}-:} \mathcal{S}^{\mathcal{D}} \longrightarrow \mathcal{S}^{\mathcal{C}}$ is a left adjoint of res ${ }_{\mathcal{D}}^{\mathcal{C}}$. Consider two diagrams $Y \in \mathcal{S}^{\mathcal{D}}$ and $Z \in \mathcal{S}^{\mathcal{C}}$. Using the adjunctions and the explicit formulas of Example 3.13 as well as Lemma 5.2 (at the second step), we have natural bijections

$$
\begin{aligned}
& \operatorname{mor}_{\mathcal{S}^{\mathcal{C}}}\left(\operatorname{mor}_{\mathcal{D}, \mathcal{C}} \underset{\mathcal{D}}{\stackrel{\mathcal{D}}{\mathbb{D}}} Y, Z\right) \cong \operatorname{mor}_{\mathcal{S} \operatorname{ets}} \mathcal{D}^{\text {op } \times \mathcal{C}}\left(\operatorname{mor}_{\mathcal{D}, \mathcal{C}}, \operatorname{map}_{\substack{\mathcal{D} \\
\underset{\mathcal{X}}{D}}}(Y, Z)\right)
\end{aligned}
$$

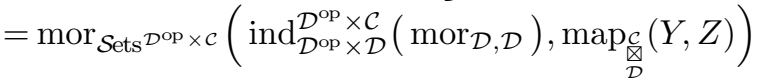

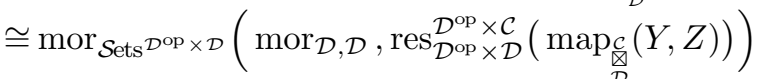

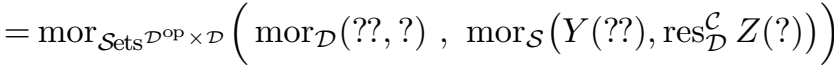

$$
\begin{aligned}
& \cong \operatorname{mor}_{\mathcal{S}^{\mathcal{D}}}\left(Y, \operatorname{res}_{\mathcal{D}}^{\mathcal{C}} Z\right) \text {. }
\end{aligned}
$$

The final bijection is an easy exercise of Yoneda style. 
For the next statements, we adopt the notations of Notation 5.1 and Lemma 5.3.

Theorem 5.4. Let $\mathcal{D}$ be a full subcategory of a small category $\mathcal{C}$, and $\mathcal{S}$ a cofibrantly generated simplicial model category with the "action" of sSets denoted by $\odot$

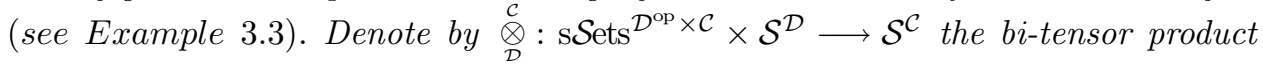
induced by $\odot$ (see Proposition-Definition 3.12 and Example 3.13$)$. In $\mathcal{U}_{\text {s }}$ ets $\left(\mathcal{D}^{\text {op }} \times\right.$ $\left.\mathcal{C}, \mathcal{D}^{\text {op }} \times \mathcal{D}\right)$, fix a cofibrant approximation

$$
\vartheta: \mathbb{E} \longrightarrow \mathbb{F}_{\mathcal{D}}^{\mathcal{C}}
$$

of the diagram $\mathbb{F}_{\mathcal{D}}^{\mathcal{C}}$ given by $\mathbb{F}_{\mathcal{D}}^{\mathcal{C}}(d, c)=\operatorname{mor}_{\mathcal{C}}(d, c)$ for all $d \in \mathcal{D}$ and $c \in \mathcal{C}$. More precisely, $\mathbb{E}$ is an arbitrary cofibrant object in this model category and $\vartheta$ is a weak equivalence $\mathcal{D}^{\text {op }} \times \mathcal{D}$-objectwise. For every diagram $X \in \mathcal{S}^{\mathcal{C}}$, we define

$$
\mathcal{Q}_{\mathcal{D}}^{\mathcal{C}} X:=\mathbb{E} \stackrel{\mathcal{D}}{\mathcal{D}}_{\operatorname{res}_{\mathcal{D}}^{\mathcal{C}}} q X
$$

and we let $\xi_{X}^{\mathcal{C}, \mathcal{D}}: \mathcal{Q}_{\mathcal{D}}^{\mathcal{C}} X \longrightarrow X$ be given by the composition

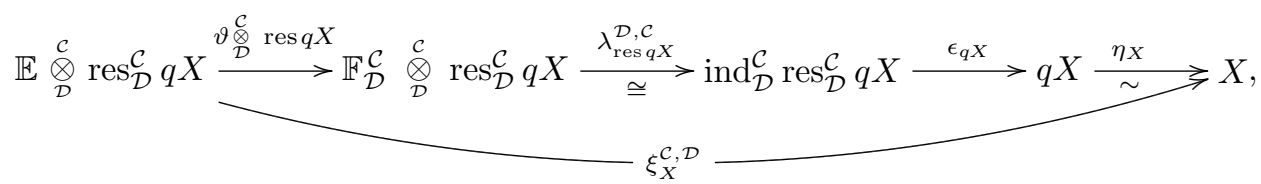

where $\epsilon_{q X}$ is the counit, at $q X$, of the adjunction $\left(\operatorname{ind}_{\mathcal{D}}^{\mathcal{C}}\right.$, $\left.\operatorname{res}_{\mathcal{D}}^{\mathcal{C}}\right)$. Then, the pair $\left(\mathcal{Q}_{\mathcal{D}}^{\mathcal{C}}, \xi^{\mathcal{C}, \mathcal{D}}\right)$ is a functorial cofibrant approximation in the model category $\mathcal{U}_{\mathcal{S}}(\mathcal{C}, \mathcal{D})$. Moreover, the morphism $\vartheta \stackrel{\mathcal{C}}{\mathcal{D}}_{\mathcal{C}} q$ is a $\mathcal{D}$-weak equivalence, $\epsilon_{q X}$ is a $\mathcal{D}$-isomorphism, and $\eta_{X}$ is a $\mathcal{C}$-weak equivalence.

After a couple of remarks and immediate corollaries, the proof will be given at the end of this section, starting with Lemma 5.9.

Remark 5.5. There is no need for $\mathbb{E}$ and $\vartheta$ to be part of a functorial cofibrant approximation: one only needs to know how to cofibrantly approximate the single diagram $\mathbb{F}_{\mathcal{D}}^{\mathcal{C}}$ in the model category $\mathcal{U}_{\text {s } \mathcal{S e t s}}\left(\mathcal{D}^{\text {op }} \times \mathcal{C}, \mathcal{D}^{\text {op }} \times \mathcal{D}\right)$. In fact, it follows from Remark 2.8 that there is a Quillen adjunction

$$
\operatorname{ind}_{\mathcal{D}^{\mathrm{op}} \times \mathcal{D}}^{\mathcal{D}_{\mathrm{o}}^{\mathrm{op}}}: \quad \mathcal{U}_{\mathrm{s} \mathcal{S} \text { ets }}\left(\mathcal{D}^{\mathrm{op}} \times \mathcal{D}\right) \rightleftarrows \mathcal{U}_{\mathrm{s} \mathcal{S e t s}}\left(\mathcal{D}^{\mathrm{op}} \times \mathcal{C}, \mathcal{D}^{\mathrm{op}} \times \mathcal{D}\right) \quad: \operatorname{res}_{\mathcal{D}^{\mathrm{op}} \times \mathcal{D}}^{\mathcal{D}^{\mathrm{op}} \times \mathcal{C}}
$$

In particular, it is enough to select a cofibrant approximation $\varrho: \mathbb{E}_{0} \longrightarrow \mathbb{F} \mathcal{D}$ in the model category $\mathcal{U}_{\text {s } S \text { ets }}\left(\mathcal{D}^{\text {op }} \times \mathcal{D}\right)$ and to induce it up to get

$$
\underbrace{\operatorname{ind}_{\mathcal{D}^{\mathrm{op}} \times \mathcal{D}}^{\mathcal{D}^{\mathrm{op}} \times \mathcal{C}}(\varrho)}_{=: \vartheta}: \underbrace{\operatorname{ind}_{\mathcal{D}^{\mathrm{op}} \times \mathcal{D}}^{\mathcal{D}_{\mathrm{op}} \times \mathcal{C}}\left(\mathbb{E}_{0}\right)}_{=: \mathbb{E}} \longrightarrow \operatorname{ind}_{\mathcal{D}^{\mathrm{op}} \times \mathcal{D}}^{\mathcal{D}_{\mathrm{op}} \times \mathcal{C}}(\mathbb{F} \mathcal{D})=\mathbb{F}_{\mathcal{D}}^{\mathcal{C}},
$$

where the latter identification is given by Lemma 5.2 ; then, $\vartheta$ and $\mathbb{E}$ fulfil the required properties needed in Theorem 5.4. The point here is that the model category $\mathcal{U}_{\text {s } S \text { ets }}\left(\mathcal{D}^{\text {op }} \times \mathcal{D}\right)$ is à la Dwyer-Kan-Heller-Dugger as considered in $[\mathbf{5}, \mathbf{6}, \mathbf{8}]$, that is, with sSets as category of "values" and without need of a relative model category structure in the sense of 2.6. See also Remark 6.12 below. 
Remark 5.6. The only property of the morphism $\eta_{X}: q X \longrightarrow X$, where $X \in \mathcal{S}^{\mathcal{C}}$, that is needed here is that it is a $\mathcal{D}$-weak equivalence of $\mathcal{C}$-indexed diagrams, depending functorially on $X$, with a $\mathcal{D}$-objectwise cofibrant source $q X$. Therefore, in this section, one could have replaced everywhere the cofibrant replacement $\left(Q_{\mathcal{S}}, \xi^{\mathcal{S}}\right)$ in the model category $\mathcal{S}$ by any functorial cofibrant approximation $\left(\mathcal{Q}_{\mathcal{S}}, \zeta^{\mathcal{S}}\right)$; the functoriality of $\zeta^{\mathcal{S}}$ guarantees that $q X$ really is a functor. One could even merely chose an arbitrary $\mathcal{D}$-objectwise cofibrant diagram $q X$ in $\mathcal{S}^{\mathcal{C}}$ and an arbitrary $\mathcal{D}$-weak equivalence $\eta_{X}: q X \longrightarrow X$ in $\mathcal{S}^{\mathcal{C}}$ at the only cost of dropping the functoriality statement in Theorem 5.4. In particular, if $X$ is already known to be $\mathcal{D}$-objectwise cofibrant, it suffices to take $q X=X$ and $\eta_{X}=\mathrm{id}_{X}$. This gives the immediate:

Corollary 5.7. Let $\mathcal{D}$ be a full subcategory of a small category $\mathcal{C}$, and $\mathcal{S}$ a cofibrantly generated simplicial model category. Fix as above a cofibrant approximation $\vartheta: \mathbb{E} \longrightarrow \mathbb{F}_{\mathcal{D}}^{\mathcal{C}}$ in the model category $\mathcal{U}_{\text {sSets }}\left(\mathcal{D}^{\text {op }} \times \mathcal{C}, \mathcal{D}^{\text {op }} \times \mathcal{D}\right)$. For every $\mathcal{C}$ objectwise cofibrant diagram $X \in \mathcal{S}^{\mathcal{C}}$, we define

$$
\mathcal{Q} X:=\mathbb{E} \stackrel{\mathcal{D}}{\mathcal{D}}_{\operatorname{res}_{\mathcal{D}}^{\mathcal{C}}} X
$$

and we let $\zeta_{X}: \mathcal{Q} X \longrightarrow X$ be given by the composition

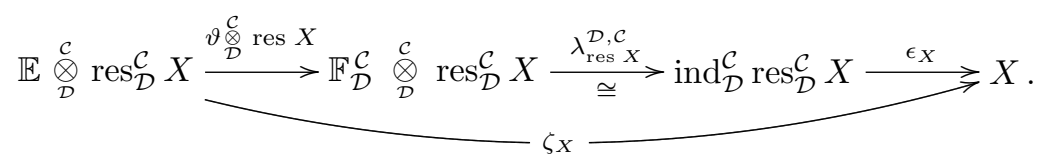

Then, $\zeta_{X}: \mathcal{Q} X \longrightarrow X$ is a cofibrant approximation in $\mathcal{U}_{\mathcal{S}}(\mathcal{C}, \mathcal{D})$.

We state the absolute case $\mathcal{D}=\mathcal{C}$ of Theorem 5.4 , using that $\operatorname{res}_{\mathcal{C}}^{\mathcal{C}}=\operatorname{id}_{\mathcal{C}}=\operatorname{ind}_{\mathcal{C}}^{\mathcal{C}}$.

Corollary 5.8. Let $\mathcal{C}$ be a small category and $\mathcal{S}$ a cofibrantly generated simplicial model category. Fix a cofibrant approximation

$$
\vartheta: \mathbb{E} \longrightarrow \mathbb{F} \mathcal{C}
$$

of the diagram $\mathbb{F} \mathcal{C}(? ?, ?)=\operatorname{mor}_{\mathcal{C}}(? ?, ?)$ in the model category $\mathcal{U}_{\mathrm{s} S \mathrm{ets}}\left(\mathcal{C}^{\mathrm{op}} \times \mathcal{C}\right)$; more precisely, $\mathbb{E}$ is a cofibrant object in the latter model category and $\vartheta$ is a weak equivalence $\mathcal{C}^{\mathrm{op}} \times \mathcal{C}$-objectwise. For every diagram $X \in \mathcal{S}^{\mathcal{C}}$, we define

$$
\mathcal{Q}_{\mathcal{C}} X:=\mathbb{E} \stackrel{\mathcal{Q}}{\mathcal{C}}_{\mathcal{C}} X
$$

and we let $\xi_{X}^{\mathcal{C}}$ be given by the composition

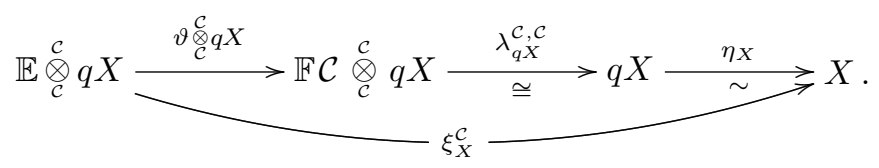

Then, the pair $\left(\mathcal{Q}_{\mathcal{C}}, \xi^{\mathcal{C}}\right)$ is a functorial cofibrant approximation in $\mathcal{U}_{\mathcal{S}}(\mathcal{C})$.

We establish now a few preparatory results for the proof of Theorem 5.4. 
Lemma 5.9. Let $\mathcal{D}$ be a subcategory of a small category $\mathcal{C}$, and let $\mathbb{E}$ be as in Theorem 5.4. Consider two objects $d \in \mathcal{D}$ and $c \in \mathcal{C}$. Then, the diagrams $\mathbb{E}(? ?, c)$ and $\mathbb{E}\left(d\right.$, ?) are cofibrant in $\mathcal{U}_{\mathrm{s} \mathcal{S} \text { ets }}\left(\mathcal{D}^{\mathrm{op}}\right)$ and $\mathcal{U}_{\mathrm{s} \mathcal{S e t s}}(\mathcal{C})$, respectively.

Proof. This is merely a special case of Remark 2.10.

Lemma 5.10. Let $\mathcal{C}$ be a small category, $\mathcal{D}$ be a subcategory of $\mathcal{C}$ and $d$ an object of $\mathcal{D}$. Then, the diagram $\mathbb{F} \mathcal{C}(? ?, d) \in \operatorname{sSets}{ }^{\mathrm{Co}}$ is cofibrant in $\mathcal{U}_{\mathrm{s} S e t s}\left(\mathcal{C}^{\mathrm{op}}, \mathcal{D}^{\mathrm{op}}\right)$.

Proof. First, recall that $\mathbb{F} \mathcal{C}(? ?, d)=\operatorname{mor}_{\mathcal{C}}(? ?, d)$. We will show that $\varnothing \longrightarrow \mathbb{F} \mathcal{C}(? ?, d)$ has the left lifting property with respect to trivial fibrations in $\mathcal{U}_{\mathrm{s} S \text { ets }}\left(\mathcal{C}^{\mathrm{op}}, \mathcal{D}^{\mathrm{op}}\right)$. Take $p: A \longrightarrow B$ a trivial fibration, i.e. a trivial fibration $\mathcal{D}$-objectwise. We only have to show that the map of sets

$$
\operatorname{mor}_{\mathcal{T}}(\mathbb{F} \mathcal{C}(? ?, d), p(? ?)): \operatorname{mor}_{\mathcal{T}}(\mathbb{F} \mathcal{C}(? ?, d), A(? ?)) \longrightarrow \operatorname{mor}_{\mathcal{T}}(\mathbb{F} \mathcal{C}(? ?, d), B(? ?))
$$

is surjective, where we abbreviate $\mathcal{T}:=\mathrm{s}_{\mathcal{S e t s}}{ }^{\mathrm{Cop}}$. We have natural bijections

$$
\operatorname{mor}_{\text {s } \mathcal{S e t s}}{ }^{\mathrm{cop}}(\mathbb{F} \mathcal{C}(? ?, d), A(? ?)) \cong \operatorname{mor}_{\mathcal{S e t s}^{\mathrm{cop}}}\left(\operatorname{mor}_{\mathcal{C}}(? ?, d), A(? ?)_{0}\right) \cong A(d)_{0},
$$

using Remark 2.2 and the Yoneda lemma. Doing the same with $B(? ?)$ and with $p(? ?)$, we are left to verify that the map $p(d)_{0}: A(d)_{0} \longrightarrow B(d)_{0}$ is surjective in $\mathcal{S}$ ets. This is immediate from the fact that $p(d)$ is a trivial Kan fibration in sSets. (This is well known and follows, for instance, from the right lifting property with respect to the cofibration $\varnothing \hookrightarrow \Delta^{0}$.)

Lemma 5.11. Let $\mathcal{D}$ be a subcategory of a small category $\mathcal{C}$, and $\mathcal{S}$ a cofibrantly generated simplicial model category. Let $\mathbb{E}$ and $\vartheta$ be as in Theorem 5.4. Suppose given $Y \in \mathcal{S}^{\mathcal{C}}$, a diagram that is $\mathcal{D}$-objectwise cofibrant. Then, the morphism

$$
\vartheta \stackrel{\mathcal{C}}{\otimes} Y: \mathbb{E} \stackrel{\mathcal{D}}{\otimes}_{\mathcal{D}}^{\mathcal{D}} Y \longrightarrow \mathbb{F}_{\mathcal{D}}^{\mathcal{C}} \stackrel{\mathcal{D}}{\otimes}_{\mathcal{D}}^{\mathcal{C}} Y
$$

is a cofibrant approximation in the model category $\mathcal{U}_{\mathcal{S}}(\mathcal{C}, \mathcal{D})$, that is, the diagram $\mathbb{E} \stackrel{\mathcal{C}}{\stackrel{\mathcal{D}}{\mathcal{D}}} Y$ is $\mathcal{D}$-cofibrant and the morphism $\vartheta \stackrel{\mathcal{C}}{\stackrel{\mathcal{D}}{C}} Y$ is a $\mathcal{D}$-weak equivalence.

Proof. Since $\mathbb{E}$ is $\mathcal{D}^{\mathrm{op}} \times \mathcal{D}$-cofibrant and $Y$ is $\mathcal{D}$-objectwise cofibrant, the diagram $\mathbb{E} \stackrel{\mathcal{C}}{\otimes} Y$ is cofibrant by Theorem 4.6 applied with $\mathcal{A}:=\mathcal{D}$. It only remains to show that $\vartheta \stackrel{\mathcal{C}}{\otimes} Y$ is a $\mathcal{D}$-weak equivalence. For all $d \in \mathcal{D}$, the morphism $(\vartheta \stackrel{\mathcal{C}}{\otimes} Y)(d)$ is equal to

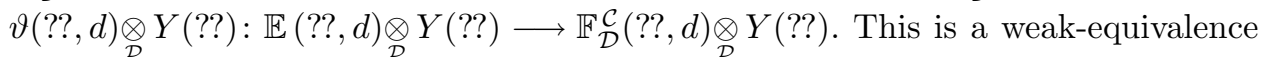
in $\mathcal{S}$ by Corollary 4.7 for $\mathcal{A}:=\mathcal{D}$ and $\xi(? ?):=\vartheta(? ?, d)$. To apply 4.7 , we need the following facts: $Y$ is objectwise cofibrant by hypothesis; the diagram $\mathbb{E}(? ?, d)$ is cofibrant in $\mathcal{U}_{\text {s } S \text { ets }}\left(\mathcal{D}^{\text {op }}\right)$ by Lemma 5.9 ; one has $\mathbb{F}_{\mathcal{D}}^{\mathcal{C}}(? ?, d)=\mathbb{F} \mathcal{D}(? ?, d)$ in sSets ${ }^{\mathcal{D}^{\text {op }}}$ and

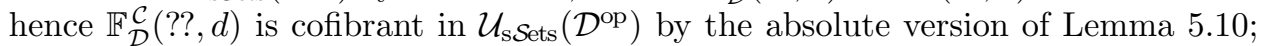
and finally $\vartheta(? ?, d): \mathbb{E}(? ?, d) \longrightarrow \mathbb{F}_{\mathcal{D}}^{\mathcal{C}}(? ?, d)$ is a weak-equivalence in $\mathcal{U}_{\text {s } S \text { ets }}\left(\mathcal{D}^{\text {op }}\right)$ by definition of $\vartheta$.

Proof of Theorem 5.4. By Lemma 5.11 applied to $Y:=\operatorname{res}_{\mathcal{D}}^{\mathcal{C}} q X$, which is $\mathcal{D}$-objectwise cofibrant, the diagram $\mathbb{E} \stackrel{\mathcal{D}}{\otimes}_{\mathcal{D}}^{\otimes} \operatorname{res}_{\mathcal{D}}^{\mathcal{C}} q X$ is $\mathcal{D}$-cofibrant and $\vartheta \underset{\mathcal{D}}{\stackrel{\mathcal{Q}}{\&}} q X$ is a $\mathcal{D}$-weak 
equivalence. Since the functor $\operatorname{res}_{\mathcal{D}}^{\mathcal{C}} \circ \operatorname{ind}_{\mathcal{D}}^{\mathcal{C}}$ is naturally equivalent to $\operatorname{id}_{\mathcal{D}}$, the morphism $\operatorname{res}_{\mathcal{D}}^{\mathcal{C}}\left(\epsilon_{q X}\right)$ is an isomorphism, that is, $\epsilon_{q X}$ is a $\mathcal{D}$-isomorphism. Since $\eta_{X}$ is a $\mathcal{D}$-weak equivalence (and even a $\mathcal{C}$-weak equivalence), $\xi_{X}^{\mathcal{C}, \mathcal{D}}$ is a $\mathcal{D}$-weak equivalence and the result follows.

\section{Explicit examples of cofibrant approximations in $\mathcal{U}_{\mathcal{S}}(\mathcal{C}, \mathcal{D})$}

In the present section, we provide an explicit cofibrant approximations of the diagram $\mathbb{F}_{\mathcal{D}}^{\mathcal{C}}$ in $\mathcal{U}_{\text {s } S \text { ets }}\left(\mathcal{D}^{\text {op }} \times \mathcal{C}, \mathcal{D}^{\text {op }} \times \mathcal{D}\right)$. With Theorem 5.4, this produces an explicit functorial cofibrant approximations in $\mathcal{U}_{\mathcal{S}}(\mathcal{C}, \mathcal{D})$.

For this section, we fix a cofibrantly generated simplicial model category $\mathcal{S}$.

We need some notations. To start with, given a small category $\mathcal{C}$, we denote by $B \mathcal{C}=B_{\bullet} \mathcal{C} \in$ s $\mathcal{S e t s}$ its nerve, whose realization $|B \mathcal{C}| \in \mathcal{T}_{\text {op }}$ is the usual classifying space of $\mathcal{C}$. Here, we follow Segal's modern definition of the nerve in [16], see also Quillen [15] (and not Bousfield-Kan's old definition in [3], where their $B \mathcal{C}$ is our $B\left(\mathcal{C}^{\mathrm{op}}\right)$; note however that $|B \mathcal{C}|$ and $\left|B\left(\mathcal{C}^{\mathrm{op}}\right)\right|$ are canonically homeomorphic).

Notation 6.1. Let $\mathcal{D}$ be a subcategory of a small category $\mathcal{C}$.

(i) Given two objects $d \in \mathcal{D}$ and $c \in \mathcal{C}$, we let $d \searrow \mathcal{D} \searrow c$ be the double-comma category with

$$
\left\{d \stackrel{\alpha}{\longrightarrow} d_{0} \stackrel{\gamma}{\longrightarrow} c \mid d_{0} \in \operatorname{obj}(\mathcal{D}), \alpha \in \operatorname{arr}(\mathcal{D}) \text { and } \gamma \in \operatorname{arr}(\mathcal{C})\right\}
$$

as set of objects, with the commutative diagrams of the form

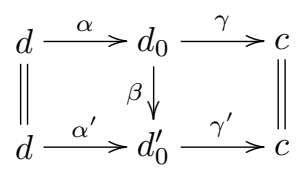

with $\beta \in \operatorname{arr}(\mathcal{D})$, as morphisms, and with the obvious concatenation of diagrams as composition. The comma category $\mathcal{D} \backslash c$ is defined similarly.

(ii) In the diagram-category $\mathrm{sSets}^{\mathcal{D}^{\mathrm{op}} \times \mathcal{C}}$, consider the diagram $\mathbb{E}_{\mathcal{D}}^{\mathcal{C}}=\mathbb{E}_{\mathcal{D}}^{\mathcal{C}}(? ?, ?)$ defined by

$$
\mathbb{E}_{\mathcal{D}}^{\mathcal{C}}:=B(? ? \searrow \mathcal{D} \backslash)^{\mathrm{op}}: \mathcal{D}^{\mathrm{op}} \times \mathcal{C} \longrightarrow \mathrm{s} \mathcal{S} \text { ets, } \quad(d, c) \longmapsto B(d \searrow \mathcal{D} \searrow c)^{\mathrm{op}}
$$

and the morphism $\vartheta_{\mathcal{D}}^{\mathcal{C}}: \mathbb{E}_{\mathcal{D}}^{\mathcal{C}} \longrightarrow \mathbb{F}_{\mathcal{D}}^{\mathcal{C}}$ given at level $n \in \mathbb{N}$ by the obvious composition, namely, for $d \in \mathcal{D}$ and $c \in \mathcal{C}$,

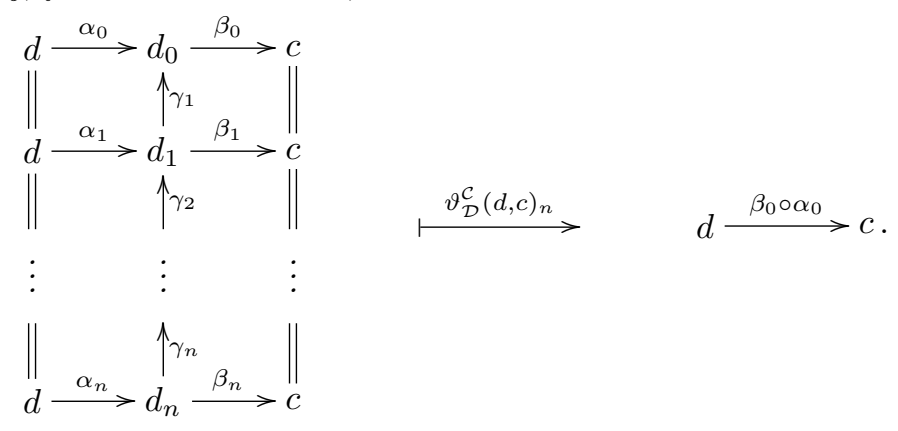


Homology, Homotopy and Applications, vol. 8(1), 2006

Note that $\beta_{j} \circ \alpha_{j}=\beta_{0} \circ \alpha_{0}$ for $0 \leqslant j \leqslant n$, so that $j=0$ plays no prominent rôle.

(iii) Define the more $\natural$ diagram ${ }^{\natural} \mathbb{E}_{\mathcal{D}}^{\mathcal{C}}={ }^{\natural} \mathbb{E}_{\mathcal{D}}^{\mathcal{C}}(? ?, ?)$ without the op's, that is,

$$
{ }^{\natural} \mathbb{E}_{\mathcal{D}}^{\mathcal{C}}:=B(? ? \searrow \mathcal{D} \searrow ?): \mathcal{D}^{\mathrm{op}} \times \mathcal{C} \longrightarrow \text { s Sets, } \quad(d, c) \longmapsto B(d \searrow \mathcal{D} \searrow c),
$$

and define the morphism ${ }^{\natural} \vartheta_{\mathcal{D}}^{\mathcal{C}}:{ }^{\natural} \mathbb{E}_{\mathcal{D}}^{\mathcal{C}} \longrightarrow \mathbb{F}_{\mathcal{D}}^{\mathcal{C}}$ similarly.

We write without decoration $\downarrow$ the diagram involving the op's since such diagrams are more commonly used, as we shall see in Section 7, for instance.

Remark 6.2. When $\mathcal{D}=\mathcal{C}$ in Notation 6.1 , for $c^{\prime}, c \in \mathcal{C}$, the category $c^{\prime} \searrow \mathcal{C} \searrow c$ is the usual double-comma category $c^{\prime} \searrow \mathcal{C} \searrow c$. In this case, we write

$$
\mathbb{E} \mathcal{C}:=\mathbb{E}_{\mathcal{C}}^{\mathcal{C}}=B(? ? \searrow \mathcal{C} \searrow ?)^{\text {op }} \quad \text { and } \quad \vartheta_{\mathcal{C}}:=\vartheta_{\mathcal{C}}^{\mathcal{C}}
$$

We define ${ }^{\natural} \mathbb{E} \mathcal{C}$ and ${ }^{\natural} \vartheta_{\mathcal{C}}$ similarly.

Remark 6.3. For a subcategory $\mathcal{D}$ of a small category $\mathcal{C}$, the diagram ${ }^{\natural} \mathbb{E}_{\mathcal{D}}^{\mathcal{C}}(? ?, ?)$ identifies canonically with the one given at level $n \in \mathbb{N}$ by

$$
\left\{? ? \stackrel{\alpha_{0}}{\longrightarrow} d_{0} \stackrel{\gamma_{1}}{\longrightarrow} d_{1} \stackrel{\gamma_{2}}{\longrightarrow} \cdots \stackrel{\gamma_{n}}{\longrightarrow} d_{n} \stackrel{\beta_{n}}{\longrightarrow} ?\right\} ;
$$

we mean that all such morphisms $\alpha_{0}$ and $\gamma_{j}$ in $\mathcal{D}$ and $\beta_{n}$ in $\mathcal{C}$ are considered (with all possible objects $d_{j}$ in $\mathcal{D}$ ), and the face maps are given by composing two successive maps and the degeneracies by inserting identities, but only among the $\gamma_{j}$ 's.

Remark 6.4. When $\mathcal{C}$ has an initial object $c_{0}$, the functor ${ }^{\natural} \mathbb{E} \mathcal{C}\left(c_{0}, ?\right)$ is canonically isomorphic to $B(\mathcal{C} \searrow$ ?) and its realization is sometimes called the "covariant classifying $\mathcal{C}$-space". If $\mathcal{C}$ has a terminal object $c_{\infty}$, then " $\mathbb{E} \mathcal{C}\left(? ?, c_{\infty}\right) \cong B(? ? \searrow \mathcal{C})$ and its realization is sometimes called the "contravariant classifying $\mathcal{C}$-space".

Theorem 6.5. Let $\mathcal{D}$ be a subcategory of a small category $\mathcal{C}$. Then, the morphism

$$
\vartheta_{\mathcal{D}}^{\mathcal{C}}: \mathbb{E}_{\mathcal{D}}^{\mathcal{C}} \longrightarrow \mathbb{F}_{\mathcal{D}}^{\mathcal{C}}
$$

is a cofibrant approximation in the model category $\mathcal{U}_{\mathrm{s} S \mathrm{Sts}}\left(\mathcal{D}^{\mathrm{op}} \times \mathcal{C}, \mathcal{D}^{\mathrm{op}} \times \mathcal{D}\right)$. One has slightly more: $\mathbb{E}_{\mathcal{D}}^{\mathcal{C}}$ is cofibrant in $\mathcal{U}_{\mathrm{s} S \text { ets }}\left(\mathcal{D}^{\mathrm{op}} \times \mathcal{C}, \mathcal{D}^{\mathrm{op}} \times \mathcal{D}\right)$ and $\vartheta$ is a weak equivalence $\mathcal{D}^{\text {op }} \times \mathcal{C}$-objectwise. The same holds for the morphism ${ }^{\natural} \vartheta_{\mathcal{D}}^{\mathcal{C}}:{ }^{\natural} \mathbb{E}_{\mathcal{D}}^{\mathcal{C}} \longrightarrow \mathbb{F}_{\mathcal{D}}^{\mathcal{C}}$.

The proof of this theorem will follow the principle of [5, Section 9] and will be presented once a few technical lemmas are established. Combined with Theorem 5.4, Theorem 6.5 provides our main explicit cofibrant approximation in $\mathcal{U}_{\mathcal{S}}(\mathcal{C}, \mathcal{D})$. We give it a name. 
Corollary-Definition 6.6. Keeping notations as in Theorems 5.4 and 6.5, for every diagram $X \in \mathcal{S}^{\mathcal{C}}$, we denote by

$$
\bar{\xi}_{X}^{\mathcal{C}, \mathcal{D}}: \bar{Q}_{\mathcal{D}}^{\mathcal{C}} X:=\mathbb{E}_{\mathcal{D}}^{\mathcal{C}} \stackrel{\mathcal{D}}{\mathcal{D}}^{\mathcal{C}} q X \longrightarrow X
$$

the corresponding functorial cofibrant approximation of $X$ in $\mathcal{U}_{\mathcal{S}}(\mathcal{C}, \mathcal{D})$. We call $\bar{Q}_{\mathcal{D}}^{\mathcal{C}} X$ the bar cofibrant approximation of $X$ in $\mathcal{U}_{\mathcal{S}}(\mathcal{C}, \mathcal{D})$. Similarly, we write

$$
{ }^{\natural} \bar{\xi}_{X}^{\mathcal{C}, \mathcal{D}}:{ }^{\natural} \bar{Q}_{\mathcal{D}}^{\mathcal{C}} X:={ }^{\natural} \mathbb{E}_{\mathcal{D}}^{\mathcal{C}} \stackrel{\mathcal{D}}{\mathcal{D}}^{\mathcal{C}} q X \longrightarrow X
$$

and call it the opposite bar cofibrant approximation of $X$ in $\mathcal{U}_{\mathcal{S}}(\mathcal{C}, \mathcal{D})$. When $\mathcal{D}=\mathcal{C}$, we also write $\left(\bar{Q}_{\mathcal{C}}, \bar{\xi}^{\mathcal{C}}\right)$ and $\left({ }^{\natural} \bar{Q}_{\mathcal{C}},{ }^{\natural} \bar{\xi}^{\mathcal{C}}\right)$. They indeed are cofibrant approximations.

Remark 6.7. Unravelling the definition of $\mathbb{E}_{\mathcal{D}}^{\mathcal{C}}$ and that of $q X$, we see that

$$
\bar{Q}_{\mathcal{D}}^{\mathcal{C}} X(?)=B(? ? \backslash \mathcal{D} \backslash ?)^{\mathrm{op}} \underset{? ? \in \mathcal{D}}{\stackrel{\mathcal{C}}{Q}} Q_{\mathcal{S}}(X(? ?)),
$$

and similarly for ${ }^{\natural} \bar{Q}_{\mathcal{D}}^{\mathcal{C}} X(?)$, but without the op's.

Remark 6.8. Since the realization of the nerve of a small category and of its opposite are homeomorphic, for the model category of compactly generated Hausdorff spaces or that of spectra of such spaces (with the strict or the stable model structure), the cofibrant approximations $\bar{Q}_{\mathcal{D}}^{\mathcal{C}}$ and ${ }^{\natural} \bar{Q}_{\mathcal{D}}^{\mathcal{C}}$ provide isomorphic diagrams.

Corollary 6.9. Keep notations as in Theorems 5.4 and 6.5. Assume that $X \in \mathcal{S}^{\mathcal{C}}$

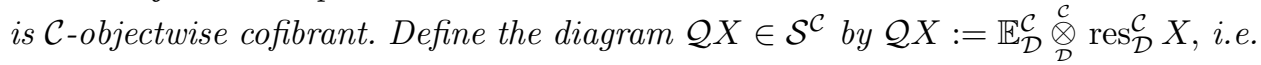

$$
\mathcal{Q} X(?)=B(? ? \backslash \mathcal{D} \backslash ?)^{\text {op }} \underset{? ? \in \mathcal{D}}{\stackrel{\mathcal{C}}{\&}} X(? ?)
$$

and the morphism $\zeta_{X}: \mathcal{Q} X \longrightarrow X$ by the composition of Corollary 5.7:

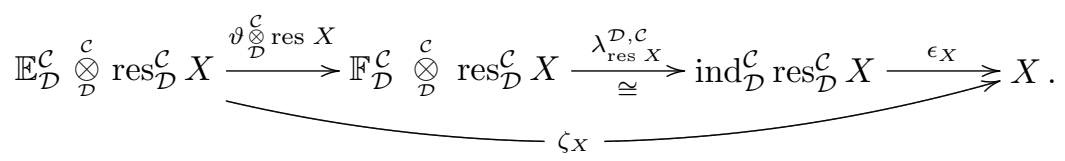

Then, $\zeta_{X}: \mathcal{Q} X \longrightarrow X$ is a cofibrant approximation of $X$ in $\mathcal{U}_{\mathcal{S}}(\mathcal{C}, \mathcal{D})$.

Proof. This follows from the above Corollary 5.7 and from Theorem 6.5.

The following two lemmas constitute preparatory material for the proof of Theorem 6.5. For the first one, recall Notation 3.8.

Lemma 6.10. Let $\mathcal{D}$ be a full subcategory of a small category $\mathcal{C}$. We have:

(i) Let $\mathcal{S}$ be a cofibrantly generated simplicial model category. If $X$ is a cofibrant object in the model category $\mathcal{U}_{\mathcal{S}}(\mathcal{C}, \mathcal{D})$, then the morphism

$$
\text { incl } \odot^{c} X: \quad \partial \Delta^{n} \odot^{c} X \longrightarrow \Delta^{n} \odot^{c} X
$$

is a cofibration in $\mathcal{U}_{\mathcal{S}}(\mathcal{C}, \mathcal{D})$ for each $n \in \mathbb{N}$. 
(ii) Let $\left\{d_{i}\right\}_{i \in I}$ a set of objects of $\mathcal{D}$. Then the diagram

$$
\coprod_{i \in I} \mathbb{F} \mathcal{C}\left(? ?, d_{i}\right) \in \mathrm{sSets}^{\mathcal{C}^{\mathrm{op}}}
$$

is a cofibrant object in the model category $\mathcal{U}_{\mathrm{s} S e t s}\left(\mathcal{C}^{\mathrm{op}}, \mathcal{D}^{\mathrm{op}}\right)$.

(iii) In a model category, an object $X$ which is a sequential colimit

$$
X=\operatorname{colim}_{n \in \mathbb{N}}\left(X_{0} \longrightarrow \cdots \longrightarrow X_{n} \longrightarrow X_{n+1} \longrightarrow \ldots\right)
$$

of cofibrations with $X_{0}$ cofibrant is itself cofibrant.

Proof.

(i) As explained in Theorem A.5, the model category $\mathcal{U}_{\mathcal{S}}(\mathcal{C}, \mathcal{D})$ inherits a canonical structure of simplicial model category with "action" given by $\odot^{\mathcal{C}}$. So, the result now follows from Remark 4.3 or [9, Proposition 9.3.9 (1) (a)] and the fact that the inclusion map incl: $\partial \Delta^{n} \longrightarrow \Delta^{n}$ is a cofibration of simplicial sets.

(ii) follows from Lemma 5.10 and the general fact that, in a model category, the class of cofibrations is determined by the left lifting property with respect to some fixed class of morphisms (trivial fibrations). This forces the coproduct, the push-out and the sequential colimit of cofibrations to be again a cofibration. This gives us (iii) as well (adding the cofibration $\varnothing \rightarrow X_{0}$ at the beginning, if one prefers).

Lemma 6.11. Let $K$ be a simplicial set. For $n \in \mathbb{N}$, denote by $\operatorname{nd}_{n}(K)$ the set of non-degenerate $n$-simplices of $K$. Then, there is a canonical isomorphism

$$
K \cong \operatorname{colim}_{n \in \mathbb{N}} \operatorname{sk}_{n}(K)
$$

of simplicial sets, where $\operatorname{sk}_{0}(K):=\Delta^{0} \times \operatorname{nd}_{0}(K)=\operatorname{nd}_{0}(K)=\underline{K_{0}}$ and for $n \geqslant 1$, $\operatorname{sk}_{n}(K)$ is determined by a push-out

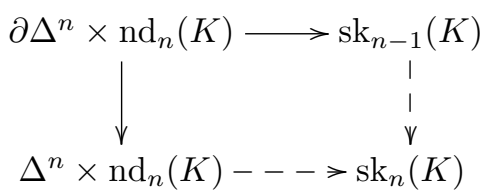

and the "structure map" $\mathrm{sk}_{n-1}(K) \longrightarrow \mathrm{sk}_{n}(K)$ for the colimit is given by the right vertical map in this push-out.

Note that $\left|\operatorname{sk}_{n}(K)\right|$ is the $n$-skeleton of $|K|$ (with its canonical CW-structure), hence the notation: see Goerss-Jardine [7, p. 8], where a proof of Lemma 6.11 can be found. 
Proof of Theorem 6.5. Let us first show that ${ }^{\natural} \vartheta_{\mathcal{D}}^{\mathcal{C}}$ is a weak equivalence. Fix two objects $d$ in $\mathcal{D}$ and $c$ in $\mathcal{C}$. We have to show that the map of simplicial sets

$$
{ }^{\natural} \vartheta_{\mathcal{D}}^{\mathcal{C}}(d, c): B(d \searrow \mathcal{D} \searrow c) \longrightarrow \underline{\operatorname{mor}_{\mathcal{D}, \mathcal{C}}(d, c)}
$$

is a weak equivalence. Consider $\operatorname{mor}_{\mathcal{D}, \mathcal{C}}(d, c)$ as a discrete category. Plainly, we have a canonical isomorphism of simplicial sets

$$
B \operatorname{mor}_{\mathcal{D}, \mathcal{C}}(d, c) \cong \operatorname{mor}_{\mathcal{D}, \mathcal{C}}(d, c),
$$

considered below as an identity and written "=". Let

$$
\pi: d \searrow \mathcal{D} \lesseqgtr c \longrightarrow \operatorname{mor}_{\mathcal{D}, \mathcal{C}}(d, c), \quad\left(d \stackrel{\alpha}{\rightarrow} d^{\prime} \stackrel{\beta}{\rightarrow} c\right) \longmapsto(d \stackrel{\beta \circ \alpha}{\longrightarrow} c)
$$

be the "composition functor" and let the "pre-insert identity" functor be

$$
\iota: \operatorname{mor}_{\mathcal{D}, \mathcal{C}}(d, c) \longrightarrow d \searrow \mathcal{D} \searrow^{c} c, \quad(d \stackrel{\gamma}{\rightarrow} c) \longmapsto\left(d \stackrel{\mathrm{id}_{d}}{\rightarrow} d \stackrel{\gamma}{\rightarrow} c\right) .
$$

Clearly, the equalities $\pi \circ \iota=\operatorname{id}_{\operatorname{mor}_{\mathcal{C}}(d, c)}$ and $B \pi={ }^{\natural} \vartheta_{\mathcal{D}}^{\mathcal{C}}(d, c)$ hold. Now, we define a natural transformation $\nu: \iota \circ \pi \longrightarrow \mathrm{id}_{\left(d \searrow \mathcal{D} \searrow_{c}^{c} c\right)}$ by the assignment

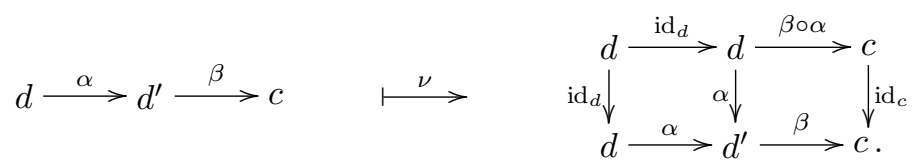

We deduce that $B \pi \circ B \iota=B(\pi \circ \iota)=B \operatorname{id}_{\operatorname{mor}_{\mathcal{C}}(d, c)}=\operatorname{id}_{\operatorname{mor}_{\mathcal{C}}(d, c)}$ and, by Segal's argument [16, Proposition 2.1], $\nu$ induces a homotopy equivalence

$$
B \iota \circ B \pi=B(\iota \circ \pi) \sim B \operatorname{id}_{\left(d \searrow \mathcal{D} \searrow_{c}^{\mathcal{C}} c\right)}=\operatorname{id}_{B\left(d \searrow \mathcal{D} \searrow_{c} c\right)},
$$

showing that $B \iota$ and $B \pi={ }^{\natural} \vartheta_{\mathcal{D}}^{\mathcal{C}}(d, c)$ are mutual inverse homotopy equivalences, hence weak equivalences.

Now, we prove that ${ }^{\natural} \mathbb{E}_{\mathcal{D}}^{\mathcal{C}}$ is a cofibrant object in $\mathcal{U}_{\text {s } S \text { ets }}\left(\mathcal{D}^{\text {op }} \times \mathcal{C}, \mathcal{D}^{\text {op }} \times \mathcal{D}\right)$. We apply Lemma $6.11 \mathcal{D}^{\text {op }} \times \mathcal{C}$-objectwise, namely, for each pair $(d, c) \in \mathcal{D}^{\text {op }} \times \mathcal{C}$, we deduce from this lemma that we have an isomorphism

$$
{ }^{\natural} \mathbb{E}_{\mathcal{D}}^{\mathcal{C}}(d, c)=B(d \searrow \mathcal{D} \searrow c) \cong \operatorname{colim}_{n \in \mathbb{N}} \operatorname{sk}_{n}\left(B\left(d \searrow \mathcal{D} \searrow^{c} c\right)\right)
$$

of simplicial sets, where

$$
\operatorname{sk}_{0}(B(d \searrow \mathcal{D} \searrow c))=\Delta^{0} \times \operatorname{nd}_{0}(B(d \searrow \mathcal{D} \searrow c))=\underline{\operatorname{obj}(d \searrow \mathcal{D} \searrow c)}
$$

and where, for each $n \geqslant 1, \mathrm{sk}_{n}$ is obtained from $\mathrm{sk}_{n-1}$ by a push-out

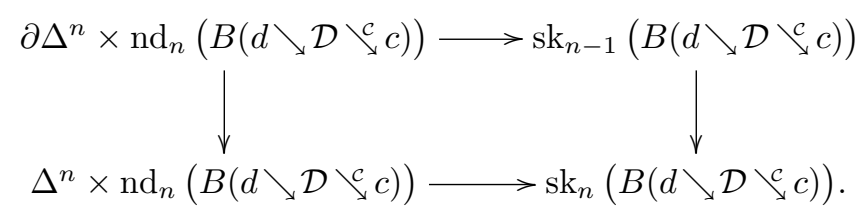

Observe from Remark 6.3 that for each $n \in \mathbb{N}$, one has a canonical bijection

$$
\operatorname{nd}_{n}(B(d \searrow \mathcal{D} \searrow c)) \cong \coprod_{\left(d_{0} \rightarrow \cdots \rightarrow d_{n}\right) \in S_{n}} \operatorname{mor}_{\mathcal{D} \times \mathcal{C}^{\mathrm{op}}}\left((d, c),\left(d_{0}, d_{n}\right)\right)
$$


where $S_{n}$ designates the set

$$
\left\{d_{0} \stackrel{\gamma_{1}}{\longrightarrow} d_{1} \stackrel{\gamma_{2}}{\longrightarrow} \ldots \stackrel{\gamma_{n}}{\longrightarrow} d_{n} \mid d_{j} \in \operatorname{obj}(\mathcal{D}) \text { and } \gamma_{i} \in \operatorname{arr}(\mathcal{D}) \text { with } \gamma_{i} \neq \operatorname{id}_{d_{i}}\right\} .
$$

Observe moreover that for a simplicial set $K$ and a set $S$, one has

$$
K \times S=K \times \underline{S}=K \odot \underline{S},
$$

where $\odot$ is as in Example 3.3 (with $\mathcal{S}$ standing for sSets) and $\underline{S}$ is "simplicially constant". So our skeleton decomposition of " $\mathbb{E}_{\mathcal{D}}^{\mathcal{C}}(d, c)=B(d \searrow \mathcal{D} \searrow c)$ becomes

$$
\operatorname{sk}_{0}\left({ }^{\natural} \mathbb{E}_{\mathcal{D}}^{\mathcal{C}}(d, c)\right):=\coprod_{d_{0} \in \mathcal{D}} \frac{\operatorname{mor}_{\mathcal{D} \times \mathcal{C}^{\mathrm{op}}}\left((d, c),\left(d_{0}, d_{0}\right)\right)}{{ }^{\prime}}
$$

and for each $n \geqslant 1$, we have a push-out square

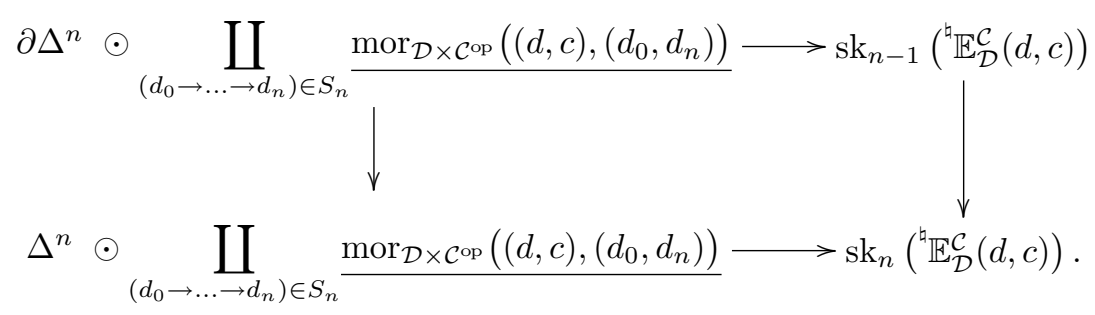

Let us rewrite the above in a more diagram-category language using Notation 5.1. We have proven that our diagram $\mathbb{E}_{\mathcal{D}}^{\mathcal{C}}(? ?, ?) \in \mathrm{sSets}^{\mathcal{D}^{\mathrm{op}} \times \mathcal{C}}$ is isomorphic to a sequential colimit

$$
\mathbb{E}_{\mathcal{D}}^{\mathcal{C}} \cong \operatorname{colim}_{n \in \mathbb{N}} \mathcal{X}_{n}
$$

of diagrams $\mathcal{X}_{n} \in$ sSets $^{\mathcal{D}^{\mathrm{op}} \times \mathcal{C}}$, where $\mathcal{X}_{n}(? ?, ?):=\operatorname{sk}_{n}(B(? ? \searrow \mathcal{D} \searrow ?))$ and we have

$$
\mathcal{X}_{0}(? ?, ?)=\coprod_{d_{0} \in \mathcal{D}} \mathbb{F}\left(\mathcal{D} \times \mathcal{C}^{\mathrm{op}}\right)\left((? ?, ?),\left(d_{0}, d_{0}\right)\right)
$$

and, for each $n \geqslant 1$, we have a push-out square (we call the left-hand map $j_{n}$ )

$$
\begin{gathered}
\partial \Delta^{n} \odot^{\mathcal{D}^{\mathrm{op}} \times \mathcal{C}} \coprod_{\left(d_{0} \rightarrow \ldots \rightarrow d_{n}\right) \in S_{n}} \mathbb{F}\left(\mathcal{D} \times \mathcal{C}^{\mathrm{op}}\right)\left((? ?, ?),\left(d_{0}, d_{n}\right)\right) \longrightarrow \mathcal{X}_{n-1}(? ?, ?) \\
\qquad=: j_{n} \\
\Delta^{n} \odot^{\mathcal{D}^{\mathrm{op}} \times \mathcal{C}} \coprod_{\left(d_{0} \rightarrow \ldots \rightarrow d_{n}\right) \in S_{n}} \mathbb{F}\left(\mathcal{D} \times \mathcal{C}^{\mathrm{op}}\right)\left((? ?, ?),\left(d_{0}, d_{n}\right)\right) \longrightarrow \mathcal{X}_{n}(? ?, ?) .
\end{gathered}
$$

We want to conclude by means of Lemma 6.10 (iii) and we have to check two things:

(a) the first object $\mathcal{X}_{0} \in$ s Sets $^{D^{\text {op }} \times \mathcal{C}}$ is cofibrant in $\mathcal{U}_{\text {sets }}\left(\mathcal{D}^{\text {op }} \times \mathcal{C}, \mathcal{D}^{\text {op }} \times \mathcal{D}\right)$;

(b) $\mathcal{X}_{n-1} \longrightarrow \mathcal{X}_{n}$ is a cofibration in $\mathcal{U}_{\text {s Sets }}\left(\mathcal{D}^{\text {op }} \times \mathcal{C}, \mathcal{D}^{\text {op }} \times \mathcal{D}\right)$ for all $n \geqslant 1$. 
Both are taken care of by Lemma 6.10. Property (a) follows from its part (ii) applied to the pair $\mathcal{D} \times \mathcal{D}^{\text {op }} \subset \mathcal{D} \times \mathcal{C}^{\text {op }}$ instead of $\mathcal{D} \subset \mathcal{C}$. The same argument guarantees that the coproduct in the above push-out square is a cofibrant object in

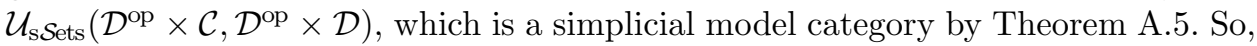
the morphism $j_{n}$ is a cofibration by part (i) of Lemma 6.10 and so is $\mathcal{X}_{n-1} \longrightarrow \mathcal{X}_{n}$ by push-out, hence (b) above. This gives the result for ${ }^{\natural} \vartheta_{\mathcal{D}}^{\mathcal{C}}:{ }^{\natural} \mathbb{E}_{\mathcal{D}}^{\mathcal{C}} \longrightarrow \mathbb{F}_{\mathcal{D}}^{\mathcal{C}}$.

The proof for $\mathbb{E}_{\mathcal{D}}^{\mathcal{C}}$ and $\vartheta_{\mathcal{D}}^{\mathcal{C}}$ is similar, mutatis op's mutandis.

Remark 6.12. Let $\mathcal{D}$ be a full subcategory of $\mathcal{C}$. With Remark 5.5 in mind, the reader might ask whether one obtains a new cofibrant approximation by the method exposed there of inducing up an approximation $\mathbb{E}_{0} \rightarrow \mathbb{F} \mathcal{D}$ from the absolute situation $\mathcal{U}_{\text {s } S \text { ets }}\left(\mathcal{D}^{\text {op }} \times \mathcal{D}\right)$ to an approximation in the relative one $\mathcal{U}_{\mathrm{s} S \text { ets }}\left(\mathcal{D}^{\text {op }} \times \mathcal{C}, \mathcal{D}^{\text {op }} \times\right.$ $\mathcal{D})$, when applied to the above $\mathbb{E}_{\mathcal{D}}^{\mathcal{C}}$. We leave it to the reader to see that nothing new happens in this way, that is, the induction of $\mathbb{E}_{\mathcal{D}}^{\mathcal{D}}$ is isomorphic to $\mathbb{E}_{\mathcal{D}}^{\mathcal{C}}$ in a compatible way with the $\vartheta$ 's, and similarly for ${ }^{\natural} \mathbb{E}$ and ${ }^{\natural} \vartheta$.

One can also wonder if other (absolute) approximations of $\mathbb{F} \mathcal{D}$, for instance, the two ones of Dugger [5], will produce essentially different approximations after induction to the relative model category. Strictly speaking the answer is yes, although inducing up the one of [5, Lemma 2.7], for instance, only differs from the above $\mathbb{E}_{\mathcal{D}}^{\mathcal{C}}$ by an "edgewise subdivision". The details are again left to the interested reader.

\section{Homotopy colimits versus colimits of cofibrant approximations}

Fix a cofibrantly generated simplicial model category $\mathcal{S}$ and a small category $\mathcal{C}$. We compare the two possible approaches to homotopy colimits. The reader should have in mind the identification $* \otimes_{\mathcal{C}}-\cong \operatorname{colim}_{\mathcal{C}}(-)$ of Example 3.10, where $* \epsilon$ $\mathrm{s} \mathcal{S}$ ets ${ }^{\mathcal{C}^{\text {op }}}$ is the constant diagram taking the point $\Delta^{0} \in \mathrm{s} \mathcal{S}$ ets as value. Comma categories are defined in Notation 6.1; for a diagram $X \in \mathcal{S}^{\mathcal{C}}$, the objectwise cofibrant approximation $q X$ and the cofibrant approximation $\bar{Q}_{\mathcal{C}} X$ are introduced in Notation 5.1 and Corollary-Definition 6.6, respectively.

Definition 7.1. Consider a diagram $X \in \mathcal{S}^{\mathcal{C}}$. Let us fix the terminology.

(i) The homotopy colimit of $X$ is the object of $\mathcal{S}$ given by

$$
\underset{\mathcal{C}}{\operatorname{hocolim}} X:=B(? ? \backslash \mathcal{C})^{\mathrm{op}} \underset{? ? \in \mathcal{C}}{\otimes} X(? ?) \text {. }
$$

(ii) The (bar) $L$-colimit of $X$ is the object of $\mathcal{S}$ given by

$$
L \operatorname{colim}_{\mathcal{C}} X:=\operatorname{colim}_{\mathcal{C}} \bar{Q}_{\mathcal{C}} X \text {. }
$$

Both constructions are clearly functorial. There are natural morphisms (in $\mathcal{S}$ )

$$
\operatorname{hocolim}_{\mathcal{C}} X=B(? ? \searrow \mathcal{C})^{\text {op }} \underset{? ? \in \mathcal{C}}{\otimes} X(? ?) \stackrel{\operatorname{Pr} \otimes \mathrm{X}}{\longrightarrow} * \underset{\mathcal{C}}{\otimes} X=\operatorname{colim}_{\mathcal{C}} X,
$$


where $\operatorname{Pr}: \mathrm{B}(? ? \backslash \mathcal{C})^{\text {op }} \longrightarrow *$ is the obvious $\mathcal{C}^{\text {op }}$-objectwise constant map, and

$$
\operatorname{Lcolim}_{\mathcal{C}} X \stackrel{\operatorname{colim}_{\mathcal{C}} \bar{\xi}_{X}^{\mathcal{C}}}{\longrightarrow} \operatorname{colim}_{\mathcal{C}} X,
$$

where $\bar{\xi}_{X}^{\mathcal{C}}: \bar{Q}_{\mathcal{C}} X \longrightarrow X$ is the morphism of 6.6 , see also Corollary 5.8.

Theorem 7.2. For every diagram $X \in \mathcal{S}^{\mathcal{C}}$, there is a commutative diagram

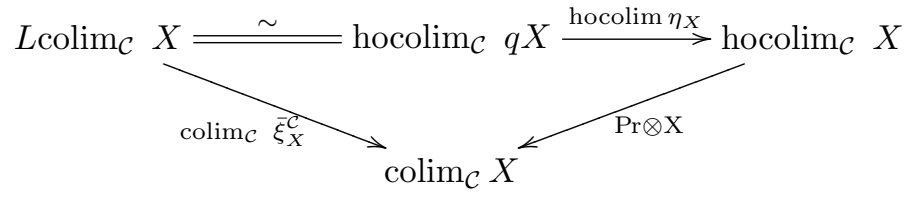

Moreover, the following properties are equivalent:

(a) The horizontal arrow $L_{\text {colim }} X \longrightarrow$ hocolim $_{\mathcal{C}} X$ is a natural weak equivalence in $\mathcal{S}$ for all $X \in \mathcal{S}^{\mathcal{C}}$

(b) The functor hocolim ${ }_{\mathcal{C}} \mathcal{S}^{\mathcal{C}} \longrightarrow \mathcal{S}$ is weakly homotopy invariant, that is, takes $\mathcal{C}$-weak equivalences to weak equivalences.

Proof. Let us start with the following two observations:

(1) The tensor product commutes with colimits, as can be checked directly from the definition or by adjunction; in particular, for every $\mathcal{X} \in \mathcal{S}^{\mathrm{Cop} \times \mathcal{C}}$ and $Y \in$ $\mathcal{S}^{\mathcal{C}}$, we have a natural isomorphism $\left(\operatorname{colim}_{\mathcal{C}} \mathcal{X}\right) \otimes_{\mathcal{C}} Y \cong \operatorname{colim}_{\mathcal{C}}\left(\mathcal{X} \stackrel{\mathcal{C}}{\mathscr{C}}^{\otimes}\right)$.

(2) We have an isomorphism $\operatorname{colim}_{? \in \mathcal{C}} B(? ? \backslash \mathcal{C} \backslash \text { ? })^{\text {op }} \cong B(? ? \backslash \mathcal{C})^{\text {op }}$. This can be checked directly from the universal property of the colimit.

We compute

$$
\begin{aligned}
& \operatorname{Lcolim}_{\mathcal{C}} X=\operatorname{colim}_{? \in \mathcal{C}}\left(\bar{Q}_{\mathcal{C}} X(?)\right) \quad \text { (by definition) } \\
& \left.=\operatorname{colim}_{? \in \mathcal{C}}\left(B(? ? \backslash \mathcal{C} \backslash ?)^{\text {op }} \underset{? ? \in \mathcal{C}}{\stackrel{\mathcal{C}}{\otimes}} q X(? ?)\right) \quad \text { (by definition of } \bar{Q}_{\mathcal{C}} X\right) \\
& \cong\left(\operatorname{colim}_{? \in \mathcal{C}} B(? ? \backslash \mathcal{C} \backslash ?)^{\mathrm{op}}\right) \underset{? ? \in \mathcal{C}}{\otimes} q X(? ?) \quad \text { (by (1) above) } \\
& \cong B(? ? \backslash \mathcal{C})^{\mathrm{op}} \underset{? ? \in \mathcal{C}}{\otimes} q X(? ?) \quad \text { (by (2) above) } \\
& =\operatorname{hocolim}_{\mathcal{C}} q X \quad \text { (by definition). }
\end{aligned}
$$

Hence the isomorphism $L \operatorname{colim}_{\mathcal{C}} X \cong \operatorname{hocolim}_{\mathcal{C}} q X$ of the statement. The commutativity of the diagram is left as an exercise. Let us see that (a) and (b) are equivalent. This is now easy. Since $L \operatorname{colim}_{\mathcal{C}}$ is a left derived functor it preserves weak equivalences, hence $(\mathrm{a}) \Longrightarrow(\mathrm{b})$. Conversely, since $\eta_{X}$ is a weak equivalence in $\mathcal{S}^{\mathcal{C}}$, one clearly deduce $(\mathrm{b}) \Longrightarrow(\mathrm{a})$ from the above.

Let us mention some important cases where the theorem applies.

Proposition 7.3. For the following (cofibrantly generated) simplicial model categories $\mathcal{S}$, the functor hocolim ${ }_{\mathcal{C}}: \mathcal{U}_{\mathcal{S}}(\mathcal{C}) \longrightarrow \mathcal{S}$ is weakly homotopy invariant, for every small category $\mathcal{C}$ : 
(i) the model category of simplicial sets;

(ii) the model category of pointed simplicial sets;

(iii) the strict model category of spectra of simplicial sets;

(iv) the stable model category of spectra of simplicial sets.

Proof. This is well known to the experts. Noting that every simplicial set and every pointed simplicial set is cofibrant (cf. [10, Proposition 3.2.2 and Corollary 3.6.6]), the result for sSets and for pointed simplicial sets follows from [9, Theorem 19.4.2 (1)] (a proof for sSets is already contained in [3, Lemma, p. 329]). For the strict model category of spectra, this follows from the case of pointed simplicial sets, since strict weak equivalences are defined levelwise and since homotopy colimits can be taken levelwise, as easily checked. For the stable model category of spectra, this is the content of [17, Lemma 5.18].

Remark 7.4. If we had defined $L$ colim using the diagram $B($ ?? $\searrow \mathcal{C} \searrow$ ?) in place of $B(? ? \backslash \mathcal{C} \searrow \text { ? })^{\text {op }}$, we would get a non-canonical zig-zag of weak equivalences instead of the canonical isomorphism in Theorem 7.2, still with a commutative diagram as in the statement. More generally, since two left derived functors of the same functor are weakly equivalent, one would have such a zig-zag of weak equivalences even if we replace $\bar{Q}_{\mathcal{C}} X$ by an arbitrary functorial cofibrant approximation of $X$.

\section{Appendix A. Simplicial model categories}

Let sSets denote the category of simplicial sets. Given two simplicial sets $K$ and $K^{\prime}$, we let $\operatorname{Map}_{\text {s } \mathcal{S e t s}}\left(K, K^{\prime}\right)=\operatorname{Map}_{\mathrm{s} \mathcal{S} \text { ets }}\left(K, K^{\prime}\right)$ • be the simplicial set defined by

$$
\operatorname{Map}_{\text {s } \mathcal{S e t s}}\left(K, K^{\prime}\right)_{q}:=\operatorname{mor}_{\mathrm{s} \mathcal{S e t s}}\left(K \times \Delta^{q}, K^{\prime}\right) \quad(q \in \mathbb{N})
$$

and with the obvious face and degeneracy maps coming from the simplicial structure of $\Delta^{q}=\operatorname{mor}_{\Delta}{ }^{\text {op }}(-,[q])$.

Definition A.1. A simplicial model category is a model category $\mathcal{M}$ equipped with

(i) a simplicial set $\operatorname{Map}\left(m_{1}, m_{2}\right)$, for all $m_{1}, m_{2} \in \mathcal{M}$;

(ii) a "composition" map of simplicial sets

$$
\operatorname{Map}\left(m_{2}, m_{3}\right) \times \operatorname{Map}\left(m_{1}, m_{2}\right) \longrightarrow \operatorname{Map}\left(m_{1}, m_{3}\right),
$$

for all $m_{1}, m_{2}, m_{3} \in \mathcal{M}$;

(iii) a bijection $\operatorname{mor}_{\mathcal{M}}\left(m_{1}, m_{2}\right) \cong \operatorname{Map}\left(m_{1}, m_{2}\right)_{0}$ (degree-zero part), that is compatible with the compositions, for all $m_{1}, m_{2} \in \mathcal{M}$.

Moreover, the following axioms are required:

(1) the "composition" map is associative with two-sided identity given, for each $m \in \mathcal{M}$, by the image of $\operatorname{id}_{m} \in \operatorname{mor}_{\mathcal{M}}(m, m)$ in $\operatorname{Map}(m, m)$; 
(2) for $K \in$ sSets and $m \in \mathcal{M}$, there exists an object $K \odot m \in \mathcal{M}$ such that, for all $\ell \in \mathcal{M}$, there is an isomorphism

$$
\operatorname{Map}(K \odot m, \ell) \cong \operatorname{Map}_{\text {sets }}(K, \operatorname{Map}(m, \ell))
$$

of simplicial sets, natural in $\ell$;

(3) for $K \in$ sSets and $m \in \mathcal{M}$, there exists an object $m^{K} \in \mathcal{M}$ such that, for all $\ell \in \mathcal{M}$, there is an isomorphism

$$
\operatorname{Map}(K \odot \ell, m) \cong \operatorname{Map}\left(\ell, m^{K}\right)
$$

of simplicial sets, natural in $\ell$;

(4) for every cofibration $p: \ell \longrightarrow \ell^{\prime}$ in $\mathcal{M}$ and for every fibration $i: m \longrightarrow m^{\prime}$ in $\mathcal{M}$, the induced map of simplicial sets

$$
\operatorname{Map}\left(\ell^{\prime}, m\right) \longrightarrow \operatorname{Map}(\ell, m) \times \operatorname{Map}\left(\ell, m^{\prime}\right) \operatorname{Map}\left(\ell^{\prime}, m^{\prime}\right)
$$

is a fibration; it is a trivial fibration if either $i$ or $p$ is moreover trivial, i.e. also a weak equivalence.

In this definition, we followed Hirschhorn [9, Section 9.1], but not Quillen [14], where he only requires axioms (2) and (3) for $K$ a finite simplicial set; as a consequence, the category $\mathcal{T}_{\text {op }}$ of all topological spaces is, for us, no simplicial model category.

Remark A.2. For a simplicial model category $\mathcal{M}$, one shows that the assignment

$$
\text { sSets } \times \mathcal{M} \stackrel{\odot}{\longrightarrow} \mathcal{M}, \quad(K, m) \longmapsto K \odot m,
$$

given by axiom (2) in the latter definition, is a (bi)functor; we will refer to it as the "action" of sSets on $\mathcal{M}$. Compare Example 3.3.

Example A.3. The model category of simplicial sets is a simplicial model category; the corresponding "action" reads $K \odot Y=K \times Y$, for $K, Y \in$ sSets. Similarly, for the category of pointed simplicial sets, we have $K \odot Y=K_{+} \wedge Y$. For the category of unpointed (resp. pointed) compactly generated Hausdorff topological spaces, the "action" is given by $K \odot Y=|K| \times Y$ (resp. $\left.K \odot Y=|K|_{+} \wedge Y\right)$.

There is an obvious asymmetry in Definition A.1 since we require the functor $(\ell, m) \mapsto \operatorname{Map}(\ell, m)$ to have what we call in Definition 4.1 the corner-map property, but we do not require the other adjoint $(K, m) \mapsto m^{K}$ to have such a property. In fact both are equivalent [9, Proposition 9.3.7].

Proposition A.4. A model category satisfying (i)-(iii) and (1)-(3) of Definition A.1 satisfies condition (4) if and only if it satisfies the following condition:

$\left(4^{\prime}\right)$ For every cofibration $i: K \rightarrow K^{\prime}$ in sSets and for every fibration $p: m \rightarrow \ell$ in $\mathcal{M}$, the induced morphism in $\mathcal{M}$

$$
m^{K^{\prime}} \longrightarrow m^{K} \times_{\ell^{K}} \ell^{K^{\prime}}
$$

is a fibration; it is a trivial fibration if either $i$ or $p$ is moreover trivial.

We use this flexibility to prove the following relative version of $[\mathbf{9}$, Theorem 11.7.3]. 
Theorem A.5. Let $\mathcal{M}$ be a cofibrantly generated simplicial model category and let $\odot: s \mathcal{S e t s} \times \mathcal{M} \longrightarrow \mathcal{M}$ be the corresponding "action" as in Remark A.2. Let $\mathcal{D} \subset \mathcal{C}$ be a pair of small categories. Then the relative model structure $\mathcal{U}_{\mathcal{M}}(\mathcal{C}, \mathcal{D})$ on $\mathcal{M}^{\mathcal{C}}$ is a simplicial model category where the "action" is given by

$$
\odot^{c}: \text { sSets } \times \mathcal{M}^{\mathcal{C}} \longrightarrow \mathcal{M}^{\mathcal{C}}, \quad(K, X) \longmapsto\left(K \odot^{c} X\right)(?)=K \odot(X(?))
$$

and where for every $K \in \mathrm{s}$ Sets and every $X, Y \in \mathcal{M}^{\mathcal{C}}$, the object $X^{K} \in \mathcal{M}^{\mathcal{C}}$ and the simplicial set $\operatorname{Map}(X, Y): \boldsymbol{\Delta}^{\mathrm{op}} \longrightarrow$ Sets are defined by

$$
X^{K}(?):=(X(?))^{K} \quad \text { and } \quad \operatorname{Map}(X, Y)_{n}=\operatorname{mor}_{\mathcal{M}^{c}}\left(\Delta^{n} \odot X, Y\right) .
$$

Proof. Hirschhorn establishes all the required adjunctions in [9, Theorem 11.7.3] and we are left to prove condition $\left(4^{\prime}\right)$ of Proposition A.4 for the relative structure, which goes as in loc. cit. Let $i: K \rightarrow K^{\prime}$ be a cofibration in the model category sSets and let $p: X \rightarrow Y$ in $\mathcal{M}^{\mathcal{C}}$ be a fibration $\mathcal{D}$-objectwise. We have to check that the induced map $\Phi: X^{K^{\prime}} \longrightarrow X^{K} \times_{Y^{K}} Y^{K^{\prime}}$ is a fibration in $\mathcal{U}_{\mathcal{M}}(\mathcal{C}, \mathcal{D})$. For any $d \in \mathcal{D}, \Phi(d)=\varphi$ where $\varphi: X^{K^{\prime}}(d)=X(d)^{K^{\prime}} \longrightarrow X(d)^{K} \times_{Y(d)}{ }^{K} Y(d)^{K^{\prime}}=$ $\left(X^{K} \times_{Y^{K}} Y^{K^{\prime}}\right)(d)$ is the corner map induced by $i: K \longrightarrow K^{\prime}$ and by $p(d): X(d) \rightarrow$ $Y(d)$, which is a fibration by assumption. The result follows from condition $\left(4^{\prime}\right)$ for $\mathcal{M}$. The same argument works if $i$ or $p$ is trivial.

\section{Acknowledgement}

This research was supported by Swiss National Science Foundation, grant 62066065.01

\section{References}

[1] P. Balmer, M. Matthey, Codescent theory I: foundations, Topology Appl. 145 (2004), pp. 11-59.

[2] P. Balmer, M. Matthey, Model theoretic reformulation of the Baum-Connes and Farrell-Jones conjectures, Adv. Math. 189 (2004), pp. 495-500.

[3] A.K. Bousfield, D.M. Kan, Homotopy Limits, Completions and Localizations, Springer Lecture Notes in Mathematics, 304, 1972.

[4] W. Chachólski, J. Scherer, Homotopy theory of diagrams, Mem. Amer. Math. Soc. 155 (2002).

[5] D. Dugger, Universal homotopy theories, Adv. Math. 164 (2001), pp. 144176.

[6] W.G. Dwyer, D.M. Kan, Function complexes for diagrams of simplicial sets, Nederl. Akad. Wetensch. Indag. Math. 45 (1983), pp. 139-147.

[7] P. Goerss, J.R. Jardine, Simplicial Homotopy Theory, Progress in Mathematics, 174, Birkhäuser, 1999.

[8] A. Heller, Homotopy theories, Mem. Amer. Math. Soc. 71 (1988).

[9] P.S. Hirschhorn, Model Categories and Their Localizations, Mathematical Surveys of the AMS, 99, 2003. 
[10] M. Hovey, Model Categories, Mathematical Surveys of the AMS, 63, 1999.

[11] D.M. Kan, On c.s.s. categories, Bol. Soc. Mat. Mexicana 2 (1957), pp. 82-94.

[12] J. Hollender, R.M. Vogt, Modules of topological spaces, applications to homotopy limits and $E_{\infty}$ structures, Arch. Math. (Basel) 59 (1992), pp. 115-129.

[13] S. Mac Lane, Categories for the Working Mathematician, GTM 5, Springer Verlag, 1971 (new edition, 1998).

[14] D. Quillen, Homotopical Algebra, Springer Lecture Notes in Mathematics, 43, 1967.

[15] D. Quillen, Higher Algebraic K-theory I. Higher K-Theories (Proc. Conf., Battelle Memorial Inst., Seattle, Washington, 1972), Lecture Notes in Mathematical, 341, Springer, Berlin, 1973, pp. 85-147.

[16] G. Segal, Classifying spaces and spectral sequences, Inst. Hautes Études Sci. Publ. Math. 34 (1968), pp. 105-112.

[17] R.W. Thomason, Algebraic K-theory and étale cohomology, Ann. Sci. Éc. Norm. Sup. 13 (1980), pp. 437-552.

Paul Balmer paul.balmer@math.ethz.ch

Department of Mathematics

ETH Zentrum

CH-8092 Zürich

Switzerland

Michel Matthey* michel.matthey@unil.ch

University of Lausanne

IGAT, EPFL

Bâtiment $\mathrm{BCH}$

CH-1015 Lausanne

Switzerland

This article is available at http://intlpress.com/HHA/v8/n1/a7/ 